\title{
Type-aware Convolutional Neural Networks for Slot Filling
}

\author{
Heike Adel \\ Hinrich Schütze \\ Center for Information and Language Processing (CIS) \\ LMU Munich, Germany
}

\author{
HEIKE.ADEL@CIS.LMU.DE \\ INQUIRIES@CISLMU.ORG
}

\begin{abstract}
The slot filling task aims at extracting answers for queries about entities from text, such as "Who founded Apple". In this paper, we focus on the relation classification component of a slot filling system. We propose type-aware convolutional neural networks to benefit from the mutual dependencies between entity and relation classification. In particular, we explore different ways of integrating the named entity types of the relation arguments into a neural network for relation classification, including a joint training and a structured prediction approach. To the best of our knowledge, this is the first study on type-aware neural networks for slot filling. The type-aware models lead to the best results of our slot filling pipeline. Joint training performs comparable to structured prediction. To understand the impact of the different components of the slot filling pipeline, we perform a recall analysis, a manual error analysis and several ablation studies. Such analyses are of particular importance to other slot filling researchers since the official slot filling evaluations only assess pipeline outputs. The analyses show that especially coreference resolution and our convolutional neural networks have a large positive impact on the final performance of the slot filling pipeline. The presented models, the source code of our system as well as our coreference resource is publicy available.
\end{abstract}

\section{Introduction}

Knowledge bases provide structured information about entities and concepts of the world. They are important resources for artificial intelligence (AI) and natural language processing (NLP) tasks, such as entity disambiguation, question answering or information retrieval (Mendes et al., 2012; Lehmann et al., 2015; Das et al., 2017). Given a knowledge base, answering a question like "Who founded Apple?" would require only a simple lookup. Similarly, an automatic assistant or dialogue system could satisfy the needs of users more easily with the access to a background knowledge base. If a user is, for example, looking for popular sights nearby, an automatic assistant could look up points of interest and information about them in a knowledge base.

Popular large-scale knowledge bases, such as Freebase (Bollacker et al., 2008) or Wikipedia (Wikipedia-URL) are often created in a large collaborative effort. Despite a lot of (manual) effort spent on their creation and maintenance, they are usually incomplete. Missing facts, however, limit their applicability in down-stream tasks. At the same time, 
there is a lot of unstructured text data available - e.g., on the internet - that mentions information missing in knowledge bases. Therefore, automatic methods for extracting structured information from text data to populate knowledge bases are important.

One specific incarnation of knowledge base population (KBP) is slot filling (Surdeanu, 2013; Surdeanu \& Ji, 2014), a shared task (Slot-filling-URL) which is yearly organized by the Text Analysis Conference (TAC). Given a large document collection and a query like "X founded Apple", the task is to extract "fillers" for the slot "X" from the document collection. The extraction of answers to the queries from large amounts of natural language text involves a variety of challenges, such as document retrieval, entity identification, coreference resolution or cross-document inferences. To cope with those challenges, most slot filling systems are pipelines of different NLP components. One of the most important components validates whether a candidate (e.g., "Steve Jobs") is a correct filler of the slot (e.g., "X" in "X founded Apple"). We take a relation classification approach to candidate validation in this paper. For example, the relation classifier validates whether the relation between the noun phrases "Steve Jobs" and "Apple" in the sentence "Steve Jobs started Apple" is the relation "X founded Y". Traditional methods to slot filling relation classification rely on (hand-crafted) patterns or linear classifiers with manually designed features. Given the variability of language, it is desirable to learn relation-specific characteristics automatically from data instead. Therefore, we design convolutional neural network architectures for the special characteristics of the slot filling task (e.g., long sentences, many inverse relations) which learn to recognize relation-specific n-gram patterns.

\subsection{Contributions}

We now describe our contributions in this paper which are centered around convolutional neural network (CNN) architectures for relation classification in the context of slot filling.

\subsubsection{Architectures and Extensive Experimentation Using Convolutional Neural Networks for Slot Filling}

We were one of the first groups to use CNNs for relation classification and demonstrate their effectiveness for slot filling (Adel, Roth, \& Schütze, 2016; Vu, Adel, Gupta, \& Schütze, 2016). The system based on this work (which is described in detail in Section 2.2) is state of the art for distantly supervised slot filling (Adel \& Schütze, 2015), see Section 4.5. In contrast to scenarios where carefully labeled gold training sets are available, relation classifiers in slot filling are trained on data that is noisy - due to error propagation through the pipeline and due to distant supervision (Adel \& Schütze, 2015; Adel et al., 2016). We show that CNNs are robust enough to be successfully applied in this noisy environment if the generic CNN architecture is adapted for relation classification and if hyperparameters are carefully tuned on a per-relation basis (see Section 3). We also show that multi-class CNNs perform better than per-relation binary CNNs in the slot filling pipeline (Section 4.3) 
probably because imposing a 1-out-of-k constraint models the data better - even though there are rare cases where more than one relation holds true.

\subsubsection{Type-Aware Relation Classification}

We define relation classification as the problem of assigning one of several relations to a 5 -tuple $\left(c_{-1}, e_{1}, c_{0}, e_{2}, c_{+1}\right)$ consisting of two entites $\left(e_{1}, e_{2}\right)$ and preceding, central and following contexts $\left(c_{-1}, c_{0}, c_{+1}\right)$. If entities are represented as they occur in the raw text, the classifier is likely to overfit to the idiosyncrasies of the entities mentioned in the training data. On the other hand, removing all entity information from the input is also harmful since entities provide valuable information for disambiguating relations; consider "Apple launches iPhone" (to start selling) vs. "SpaceX launches Falcon 9" (to send into orbit). A major focus of this work is type-aware relation classification, a middle ground between complete entity information and no entity information: only the predicted types of the entities are made available. Type information is arguably the key information needed for disambiguation in relation classification - e.g., this is the case for our "launch" example); and it prevents overfitting to entity idiosyncrasies. We design three type-aware architectures: a simple pipeline of first type classification and then relation classification (see Section 3.3.1); a joint model (Section 3.3.2); and a structured prediction model that more directly takes into account the dependencies between entity and relation classes (Section 3.3.3). In our experiments in Section 4.3, we show that the structured prediction model outperforms the other models in terms of macro $F_{1}$, the best measure of performance for difficult cases because it gives equal weight to rare and frequent relations.

\subsubsection{ANALYSIS}

The TAC KBP organizers only evaluate the final results of the entire slot filling pipeline. We perform an extensive and detailed analysis (Sections 5.1 and 5.2) and several ablation studies (Section 5.3) on individual modules of the pipeline. Inter alia, we quantify the impact of entity linking, coreference resolution and type-aware CNNs on the overall pipeline performance. We hope that this will be of great benefit to the community because this analysis - in contrast to the official TAC KBP evaluation - allows researchers to assess the impact of individual components and which components are worth investing more research effort in.

\subsubsection{RESOURCES}

We make our complete slot filling system, including the source code, publicly available at http://cistern.cis.lmu.de/CIS_SlotFilling. Since slot filling poses many NLP challenges, building such a system is a substantial software development and research effort. Through publication of the system, we share our experience with the community and lower the barriers to entry for researchers wishing to work on slot filling. 
The component of our pipeline that has the longest runtime - a runtime of several months on the entire TAC source corpus - is coreference resolution. Therefore, we publish the output of the coreference resolver (Stanford CoRENlp by Manning et al., 2014) for the two million documents of the TAC source corpus at http://cistern.cis.lmu.de/ corefresources. It consists of 198 million mentions linked in 54 million coreference chains. This will make it easier for other researchers to take advantage of coreference resolution in their systems.

By making these resources available to the NLP community, we aim to promote research in knowledge base population in general and slot filling in particular.

\subsection{Relation to Our Prior Publications}

In this subsection, we delineate our contributions in this paper from our prior publications.

In earlier work (Adel \& Schütze, 2015; Adel et al., 2016), we compared binary convolutional neural networks to traditional models for slot filling (patterns and support vector machines). Binary models facilitate extensions of the slot list with a few more slots since new models can be trained for the new slots but the existing models do not need to be retrained. However, the more slots there are, the more models need to be optimized, maintained and evaluated. Therefore, we explore multi-class convolutional neural networks for slot filling in this paper. In our experiments, we compare the novel multi-class models to the previously trained binary models.

The contribution of type-aware models which lead to our best results on the official slot filling evaluation data, is entirely novel to this paper. Although entity types are wellstudied features for traditional slot filling models (e.g., Angeli et al., 2014a; Kisiel et al., 2014), this is the first work to explore end-to-end type-aware neural networks for slot filling and show their positive impact in the pipeline setting.

Our three approaches for type-aware neural networks build on models we proposed earlier (Yaghoobzadeh, Adel, \& Schütze, 2017; Adel \& Schütze, 2017). In contrast to these two prior studies, we adapt the architectures of the models to the requirements of the slot filling task, e.g., making them more robust against unknown rare test entities and against the existence of inverse relations. This is also the first work to evaluate the CNNs with structured prediction in a noisy scenario which is arguably conceptually different to both clean data with manual annotations and distantly supervised data used without pipelines. For structured prediction, we formalize the task of joint entity and relation classification as a triple of predictions (similar to a knowledge base triple) which enables the model to learn which entity and relation classes often co-occur together. This is a novelty to slot filling which has been approached mainly with pattern matching or classification so far.

\subsection{Structure}

The remainder of the paper is organized as follows. First, the slot filling task and its challenges are described (Section 2.1). Section 2.2 presents our slot filling system which 
we used in the official shared task competition in 2015. In Section 3, we describe our convolutional neural network for slot filling relation classification and introduce multi-class models as well as models for the joint task of entity and relation classification. Afterwards, we present our experiments and discuss our results in Section 4 . Section 5 provides the results of a recall analysis, a manual categorization of the errors of our system and several ablation studies. Section 6 presents related work. Finally, Section 7 concludes the paper.

\section{Slot Filling}

In this section, we describe the slot filling task and its challenges and present the most important aspects of our slot filling system.

\subsection{Task and Challenges}

The TAC KBP slot filling task addresses the challenge of gathering information about entities (persons, organizations or geo-political entities) from a large amount of unstructured text data (Surdeanu, 2013; Surdeanu \& Ji, 2014). The input is a query similar to the following one (fictional query with a random query id and document id):

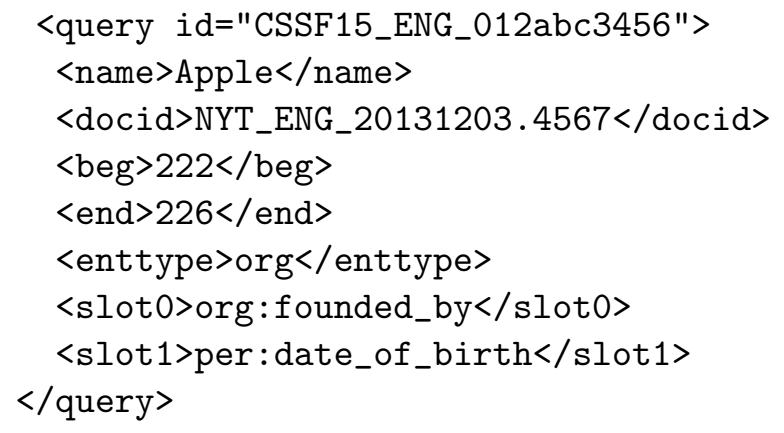

This sample query asks for the founders of the company Apple as well as for their dates of birth. It consists of a unique identifier (query id), the name of an entity (name), which we will call query entity in the following, and the type of this entity (enttype) which can be either person, organization or geo-political entity. Furthermore, it contains the slots to be filled (slot0, slot1), i.e., the questions that should be answered for the query entity, as well as a starting point in the document collection (docid with begin (beg) and end offset (end)) which points to a mention of the query entity. The starting point usually does not provide the answer to the query but it can be used to disambiguate different entities with the same name.

Figure 1 illustrates the slot filling task which is described in the following. The system has access to a large document collection which needs to be processed in order to answer the query - in the example, to find the founders of Apple and their dates of birth. The query can be split into two parts: First, the question posed by sloto ("Who are the founders of 


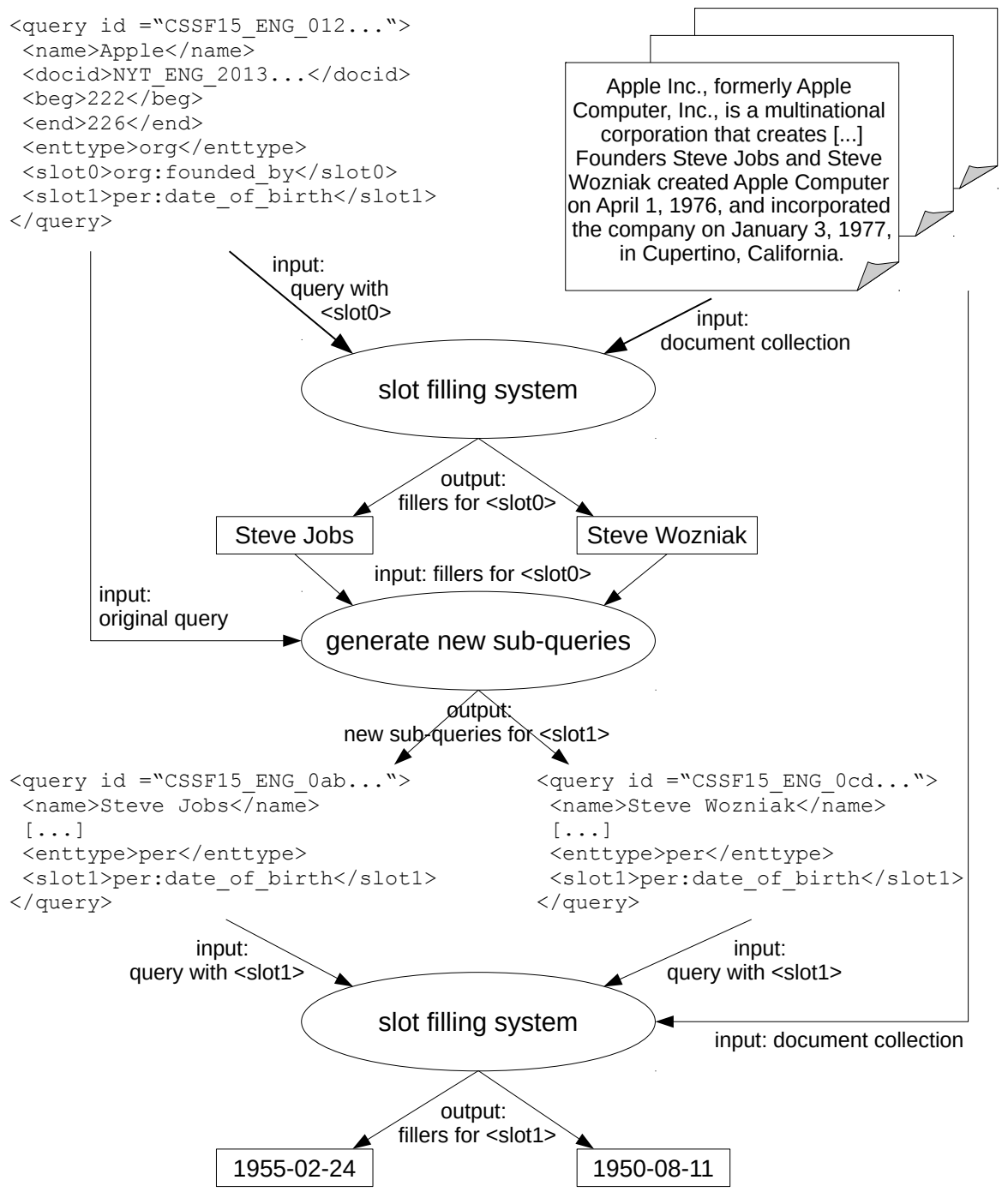

Figure 1: Overview of the slot filling task with a multiple-hop query.

Apple?") needs to be answered. Based on the results of the system on this slot, slot1 is processed ("What is their date of birth?"). Since this corresponds to taking a hop in the corresponding knowledge graph (from Apple to its founders to their date of birth), a query with two slots is also called multiple-hop query, and the question posed by sloto is called hop 0 and the question posed by slot1 is called hop 1 . The shared task organizers provide a script to generate new sub-queries for hop 1 given the system results for hop 0 and the original set of queries. The output of the system should contain the answer for each given slot (e.g., "Steve Jobs" and "Steve Wozniak" for org:founded_by), a supporting sentence 
from the document collection (e.g., "Founders Steve Jobs and Steve Wozniak created Apple Computers on April 1, 1976") as well as a confidence score. The slots can be single-valued (for instance, per:date_of_birth: a person has only one date of birth) or list-valued (for instance, org:founded_by: a company might have more than one founder). In total, there are 65 slots, out of which 18 are single-valued and 47 are list-valued. The answer of the slot filling system is assessed as correct if both the slot filler and the supporting sentence are correct. In the official evaluations, human annotators assess system outputs manually. Based on these assessments, individual results for both hops are reported as well as an overall result.

Previous work on slot filling showed that this task includes a variety of NLP challenges (Pink, Nothman, \& Curran, 2014; Min \& Grishman, 2012; Surdeanu \& Ji, 2014), such as alternate names for the same entity, ambiguous names (i.e., the same name for different entities), misspellings, coreference resolution, location inference, cross-document inference and relation extraction / classification. Our slot filling system addresses most of these challenges (except for cross-document inference which we only consider in the context of location inference).

\subsection{The CIS Slot Filling System}

For filling slots for persons, organizations and geo-political entities, i.e., for answering the questions posed by the input queries, a variety of natural language processing steps need to be performed. Our system addresses the slot filling task in a modular way. This has several advantages, including extensibility, component-wise analyzability (see Section 5.2) and modular development. In this section, an overview of the different components of our system is given. They are also depicted in Figure 2. More details can be found in our shared task system description paper (Adel \& Schütze, 2015).

\subsubsection{Alias component}

The alias component expands the query with possible aliases for the entity name. For this purpose, we employ a preprocessed list of possible aliases based on Wikipedia redirects which we extracted using JWPL (Ferschke, Zesch, \& Gurevych, 2011) on a Wikipedia dump from July 2014. If the query entity is an organization, we also add various companyspecific suffixes to the list of aliases, such as "Corp", "Co", "Inc". If the query entity is a person, we include nicknames taken from the web (Nicknames-URL) into the list of aliases.

\subsubsection{INFORMATION RETRIEVAL COMPONENT}

Based on the entity name (and aliases), documents mentioning this name are retrieved to reduce the large search space. For this, we apply the open-source system TERRIER (Ounis et al., 2006) with the following set of queries:

- AND combination of the tokens of the entity name as given in the input query 


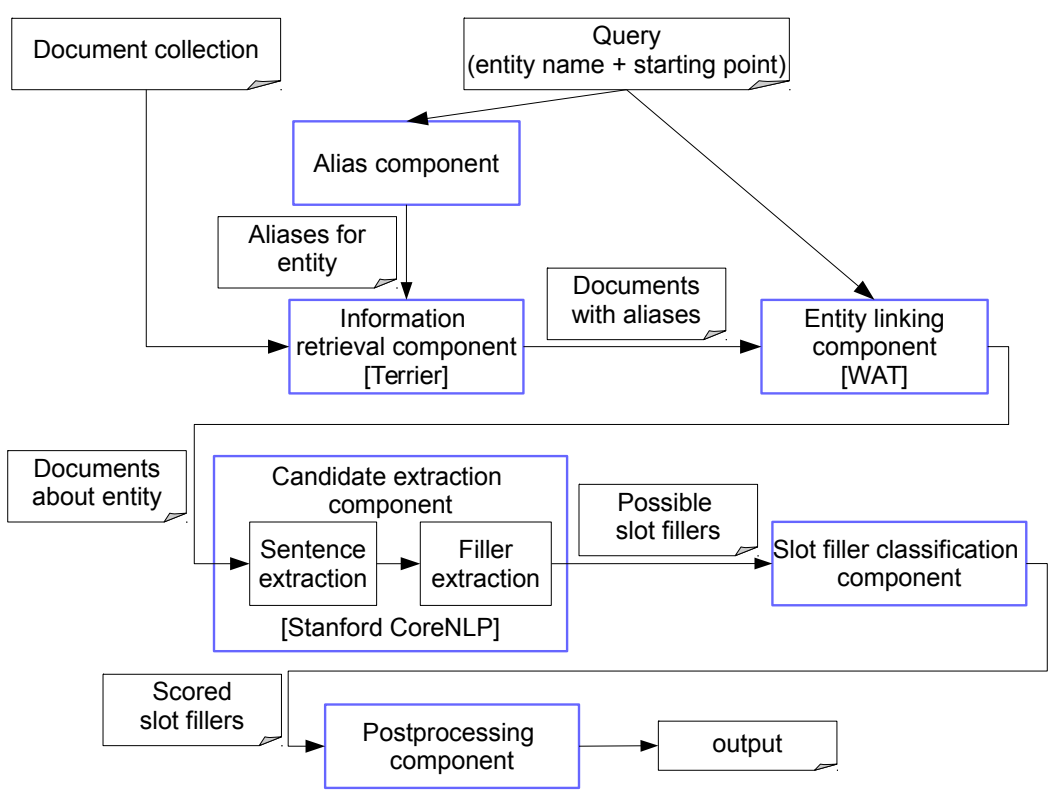

Figure 2: System overview: Basic components of the CIS slot filling system.

- AND combination of the tokens of an alias

- OR combination of the tokens of the entity name as given in the input query

For geo-political entities, we only use the two AND queries. In prior experiments, we also investigated phrase queries but found that they did not work well with spelling variations, resulting in a considerably lower overall recall of the system. Instead, we filter the resulting list of relevant documents by fuzzy string matching with the name and aliases to skip documents mentioning both the first and the last name of a person but not in a phrase.

For each entity, the results of the subqueries are ordered according to the relevance score assigned by TERRIER, concatenated and limited to the top 300 documents.

\subsubsection{ENTITY LINKING COMPONENT}

For disambiguating entities with the same name, we apply the entity linking system WAT (Piccinno \& Ferragina, 2014). It takes a sentence as input and determines the Wikipedia id of every entity in that sentence. In order to get the Wikipedia id of the query entity, we apply it to the sentence specified by the starting point of the query. Afterwards, we check for each document returned by the information retrieval component whether the mention in the document which matches the name of the query entity refers to the same Wikipedia entity as the query, i.e., whether WAT assigns it the same Wikipedia id as the query entity. In the case of a mismatch, the document is ignored in the following steps. 
After entity linking, we limit the set of documents to the 100 documents with the highest relevance score according to TERRIER. This number has been determined empirically in prior experiments: On data from previous slot filling evaluations (2013 and 2014), we observed that 100 documents are a good trade-off between recall and processing time.

\subsubsection{CANDidate EXtraction COMPOnENT}

From the remaining documents, possible slot fillers (filler candidates) are extracted.

Genre-Specific Document Processing. First, the documents are split into sentences (with Stanford CoreNlP) and cleaned, i.a., by removing html tags. Since the TAC 2015 evaluation corpus consists of two genres (news and discussion forums), our document processing and cleaning steps are genre-dependent. Prior analysis showed that this is crucial to reduce the noise in the input to the following pipeline components.

Coreference Resolution. Second, fuzzy string matching (based on Levenshtein distance) and automatic coreference resolution (with Stanford CORENLP) is performed in order to retrieve sentences mentioning the query entity.

Different studies show the importance of coreference resolution for slot filling (Min \& Grishman, 2012; Pink et al., 2014; Surdeanu \& Ji, 2014). While most systems apply coreference resolution only for matching the query entity, we also use it for the filler candidates if the named entity type of the filler is PERSON. This improves the recall of the system considerably (e.g., consider the slot org: students and the sentence "He went to University of Munich"). In Section 5.3.2, we show the positive impact of coreference resolution on the slot filling pipeline results.

Given only raw text data, the runtime of the slot filling system is mainly determined by coreference resolution. Therefore, we preprocessed the TAC source corpus (which consists of over two million documents) and stored the coreference information. In total, we have extracted about 54M coreference chains with a total number of about $198 \mathrm{M}$ mentions. The processing of all documents of the source corpus takes a considerable amount of time and may be infeasible in case of restricted computational resources. Therefore, we make this resource publicly available to the community (see Section 1.1).

Filler Candidate Extraction. Given sentences with mentions of the query entity, the system extracts possible filler candidates based on named entity tags. For named entity tagging, we apply CORENLP. For example, for the slot per:date_of_birth, the system would only consider dates as filler candidates while for a slot like org:members, the fillers can be organizations, locations or persons. For slots with string fillers, such as per:title or per: charges, we have automatically assembled lists of possible filler values from Freebase (Bollacker et al., 2008). The lists have been cleaned manually in order to improve their precision. 


\subsubsection{Slot Filler Classification Component}

The classification component identifies valid fillers for the given slot based on the textual context of the extracted filler candidates. This is mainly a relation classification task with the additional challenges that no designated training data is available and that the classifier inputs are the results from previous pipeline steps and can, thus, be noisy (e.g., due to wrong coreference resolution, wrong named entity typing or erroneous sentence splitting). Given our previous results (Adel et al., 2016), we combine traditional models (patterns and support vector machines (SVMs), similar to the ones used by Roth et al., 2013) with convolutional neural networks (CNNs) in this component. The weights for combining the model scores are tuned on previous slot filling evaluation data. Section 3 describes the CNNs in more detail.

\subsubsection{Postprocessing Component}

As a last step, the filler candidates are postprocessed and output along with the confidence scores from the classification component and the contexts they appear in.

Output Thresholds and Ranking. Based on the classification scores, filler candidates are selected for output or discarded. This decision is done based on slot-specific thresholds which were tuned on previous evaluation data. For the second slot (hop 1) of multiple-hop queries, we increase the thresholds by 0.1 in order to reduce false positive answers. (An answer to a hop 1 sub-query is only scored as correct if both the hop 0 answer and the hop 1 answer are correct. Thus, errors are propagated from hop 0 to hop 1.) The selected filler candidates are ranked according to their classification score. For single-valued slots, only the top filler candidate is output. For list-valued slots, the top $N$ filler candidates are output. ( $N$ is slot-dependent and has been determined heuristically on previous evaluation data in order to increase the precision of the system.)

Location Disambiguation and Inference. As we will describe below in Section 3.1, we do not distinguish between cities, states-or-provinces, and countries in the classification component. Before outputting the results, however, the extracted locations need to be disambiguated. The system uses city-, state- and country lists from Freebase, Wikipedia (State-list-URL) and an online list of countries (Country-list-URL) to decide to which category a location belongs. If the system extracted a city or state while the slot given in the query is a state or country, the system automatically infers the answer for the desired slot based on city-to-state, city-to-country and state-to-country mappings extracted from Freebase.

\section{Convolutional Neural Networks for Slot Filling Relation Classification}

Convolutional neural networks (CNNs) have been applied successfully to natural language processing in general (Collobert et al., 2011; Kalchbrenner, Grefenstette, \& Blunsom, 2014) 
and relation classification in particular (Zeng et al., 2014; dos Santos, Xiang, \& Zhou, 2015; $\mathrm{Vu}$ et al., 2016). We integrate them into a slot filling pipeline. This poses two additional challenges to them which prior work usually does not consider in combination: noisy labels at training time due to distantly supervised training data; and noisy or wrong inputs at test time due to error propagations in the slot filling pipeline. Examples for the latter are wrong sentence boundaries (resulting in incomplete or very long inputs), wrong coreference resolution or wrong named entity tags (resulting in incorrect candidate entites for relation classification). Our results show that CNNs are still able to classify the relations and improve the final performance of the system.

There are three reasons why CNNs are promising models for slot filling relation classification: (i) Convolutional filters of length $n$ automatically create features for every possible $n$-gram in the sentence. Since relation-indicative phrases are often $n$-grams (examples: "was born in" or "subsidiary of"), each convolutional filter can learn to recognize a particular $n$-gram and assign a high score to it. (ii) Max pooling, i.e., only considering the highest activations from each filter application result, helps extracting the most relevant $n$-grams independent of their position in the sentence. Thus, the following network layers can focus on those most relevant parts of the sentence only. (iii) The representation of input words with word embeddings and the internal computation of phrase and sentence representations based on them enables the network to recognize words or phrases which are similar to the ones seen during training. Thus, if a convolutional filter has learned to assign high scores to the $n$-gram "was founded by" during training, it can also recognize a phrase like "was established by" during testing even if this phrase did not occur in the training data (assuming that the embeddings of "founded" and "established" are similar). This is an advantage of neural models compared to pattern matching or bag-of-word approaches for which this generalization is more difficult.

\subsection{General Remarks}

For training and evaluating the convolutional neural networks, we replace the query entity with the tag <name $\rangle$ and the candidate filler with the tag $\langle$ filler $\rangle$. This prevents the model from remembering entities from the training data and helps it to focus on the context words instead. Furthermore, we only train one model for each slot and its inverse, for example per:parents and per:children. For this, we transform all training examples of per:parents into training examples for per:children by reversing the <name> and $<$ filler $>$ tags in the sentences. This avoids redundant training and helps the model to discriminate between the two inverse slots. To extract the probability for an inverse slot during test time (e.g., for per:parents), we again reverse the <name $\rangle$ and $\langle$ filler $\rangle$ tags of the sentence and extract the probability for the corresponding slot (e.g., per:children). We also merge the "city", "country" and "state-or-province" slots to one "location" slot since we expect their fillers to appear in very similar contexts. 
ADEL \& SCHÜTZE

\subsection{Basic Architecture}

This section describes the basic architecture of our convolutional neural network for slot filling relation classification (without entity types).

\subsubsection{MODEL InPuT}

We only use features which are directly available from the input context, i.e., words and combinations of words, but no hand-crafted features, such as part-of-speech tags or dependency paths. One reason for that is to avoid potential noise in the inputs due to wrong tags or wrong dependency paths. As described before, the slot filling pipeline introduces different kinds of noise anyway. Therefore, we aim for limiting additional noise as much as possible. Another reason is previous results: In earlier work (Adel et al., 2016), we showed that models without dependency paths as features are able to outperform models using dependency paths (Mintz ++ and MimLRE) on slot filling relation classification. Similarly, Roth et al. (2013) achieved the best results in the slot filling evaluations with only a minimalistic feature set.

We split the input sentence into three parts: left of the candidate relation arguments (left context), between the arguments (middle context) and right of them (right context). These three contexts form the input to the CNN, together with a flag indicating whether the query entity or the candidate filler appears first in the sentence. This flag is important for disambiguating inverse relations (see Section 3.1).

\subsubsection{Convolutional Layer}

The words of the input sentence are represented with word embeddings pre-trained with word2vec (Mikolov et al., 2013) on English Wikipedia. As described in Section 3.2.1, the input sentence is split into three parts: left, middle and right context. The network applies the following Equations 1-6 for convolution and 3-max pooling (Kalchbrenner et al., 2014) to each of these three parts individually but with convolutional filters $H$ and bias terms $b$ shared across the three contexts.

$$
\begin{aligned}
C_{\text {left }} & =\tanh \left(I_{\text {left }} * H+b\right) \\
C_{\text {middle }} & =\tanh \left(I_{\text {middle }} * H+b\right) \\
C_{\text {right }} & =\tanh \left(I_{\text {right }} * H+b\right)
\end{aligned}
$$

The symbol $*$ denotes convolution, $I$ is an input context, $H$ is the filter matrix and $b$ the bias term. We apply Equations 1-3 with multiple filter matrices $H$. The number of filter matrices $m \in\{100,300,1000\}$ is tuned on the development set. After convolution, 3-max pooling is applied which extracts the three maximum values of each $C$ (in the same order 
as they appeared in the input sequence), yielding the pooling results $P_{\text {left }}, P_{\text {middle }}, P_{\text {right }}$ :

$$
\begin{aligned}
P_{\text {left }}[i] & =\left[C_{\text {left }}[i, t] \mid R_{t}\left(C_{\text {left }}[i, t]\right) \leq 3\right] \\
P_{\text {middle }}[i] & =\left[C_{\text {middle }}[i, t] \mid R_{t}\left(C_{\text {middle }}[i, t]\right) \leq 3\right] \\
P_{\text {right }}[i] & =\left[C_{\text {right }}[i, t] \mid R_{t}\left(C_{\text {right }}[i, t]\right) \leq 3\right]
\end{aligned}
$$

with $[i]$ denoting the $i$-th row and $[i, t]$ the cell in the $i$-th row and $t$-th column of a matrix. The sequence $[p \mid P(p)]$ contains all elements $p$ that satisfy predicate $P$, in this case all elements whose rank $R_{t}$ along the time axis $t$ is 1,2 or 3 . For more details on k-max pooling, see Kalchbrenner et al. (2014).

Because of convolution and pooling, the network is able to recognize relevant $n$-grams independent of their position in the input sentence. Afterwards, the results are concatenated to one large vector and extended with a flag $v$ indicating whether the entity or the filler candidate appears first in the sentence. The final vector is passed to a multi-layer perceptron with one hidden layer.

$$
s=\tanh \left(W_{1}^{\top} P_{\text {left }}+W_{2}^{\top} P_{\text {middle }}+W_{3}^{\top} P_{\text {right }}+W_{4}^{\top} v+d\right)
$$

The matrices $W_{1}, W_{2}, W_{3}$ and $W_{4}$ are the weights of the hidden layer, $d$ is its bias term.

\subsubsection{Output LAYER}

Finally, a softmax layer is applied to the sentence representation $s$. In earlier work (Adel \& Schütze, 2015; Adel et al., 2016), we only trained binary models which output 1 if $s$ expresses the given slot or 0 if it does not. In this paper, we explore multi-class models. In the case of multi-class models, the output vector contains one output entry for each slot (except for inverse slots, see Section 3.1).

Figure 3 depicts the structure of the CNN.

\subsection{Type-aware Convolutional Neural Networks}

The relation arguments of the input for the binary models all have named entity types corresponding to the expected types of the slots. The model for the relation per:date_of_birth, for example, only gets sentences with one relation argument being a PERSON and the other relation argument being a DATE. In our training data, this is ensured by the design of the extraction process of positive and negative examples (see Section 4.1.1). In the slot filling pipeline, the candidate extraction component extracts filler candidates based on their named entity types. In contrast to the input of the binary models, the input of the multiclass model can contain relation arguments of all available types. This complicates the relation classification task of the model. A context for the relation per:date_of_birth, for example, might be similar to a context for the relation per:location_of_birth. To simplify the classification, we propose to provide the models with the named entity types of 


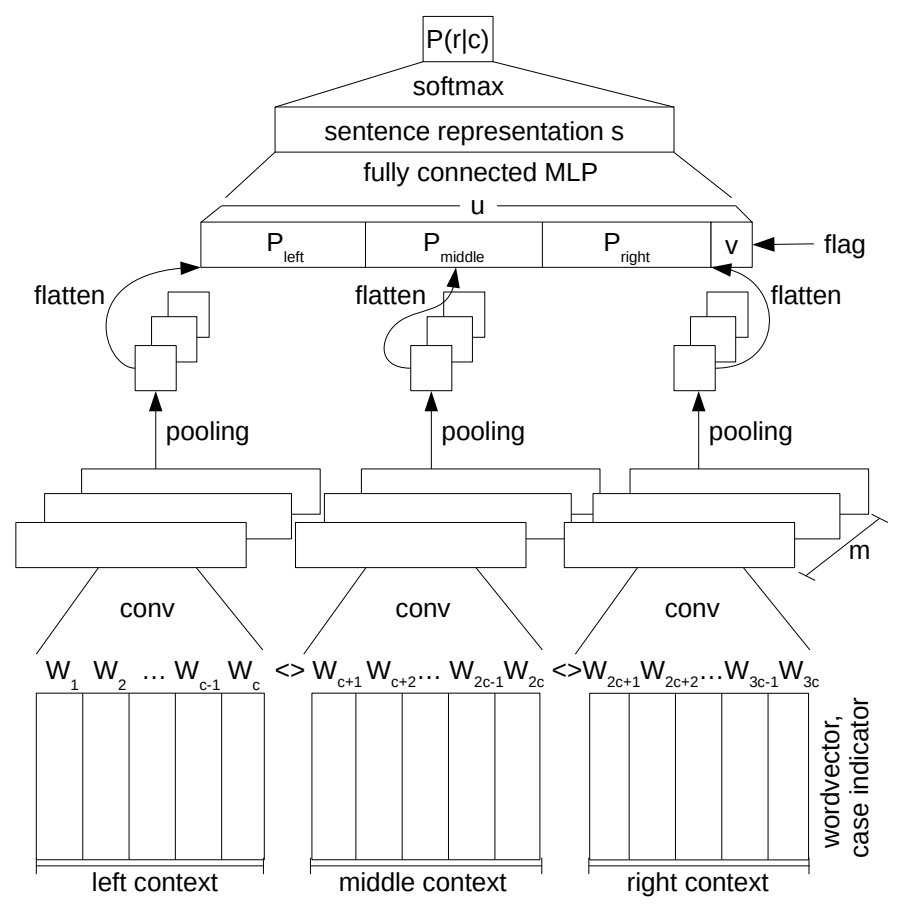

Figure 3: Convolutional neural network for slot filling.

the relation arguments. In particular, we investigate three different settings for augmenting the input of the multi-class model with named entity types. They are described in the following paragraphs. For all settings, we use the same coarse-grained types as we process in the slot filling pipeline, namely PERSON, ORGANIZATION, LOCATION, DATE, NUMBER, 0 .

\subsubsection{Pipeline Approach}

The first approach we explore is a pipeline approach based on the slot to evaluate. We use two binary (one-hot) type vectors $t_{1}$ and $t_{2}$ of the size of the type vocabulary as additional input to the network from Figure 3. For the slot per: employee_or member_of, for example, the type vector $t_{1}$ for the first relation argument would consist of only one 1 at the position of PERSON and 0 otherwise. The type vector $t_{2}$ for the second relation argument would consist of a 1 at the position of ORGANIZATION and a 1 at the position of LOCATION since a person can be employed by an organization or by a geo-political entity. Since the type vectors are based on the slots and the filler extraction with named entity recognition (see Section 2.2.4), we call this approach "pipeline". The type vectors are then fed into a hidden layer which creates type embeddings $E_{1}$ and $E_{2}$ : 


$$
E_{i}=\tanh \left(V^{\top} t_{i}+c\right)
$$

with $V$ being the weight matrix and $c$ the bias term of the hidden layer.

Then, we concatenate the type embeddings $E_{1}$ and $E_{2}$ with the pooling results of the $\mathrm{CNN}$ for relation classification to calculate a type-aware sentence representation $s$. Thus, Equation 7 becomes:

$$
s=\tanh \left(W_{1}^{\top} P_{\text {left }}+W_{2}^{\top} P_{\text {middle }}+W_{3}^{\top} P_{\text {right }}+W_{4}^{\top} v+W_{5}^{\top} E_{1}+W_{6}^{\top} E_{2}+d\right)
$$

This is depicted in Figure 4.

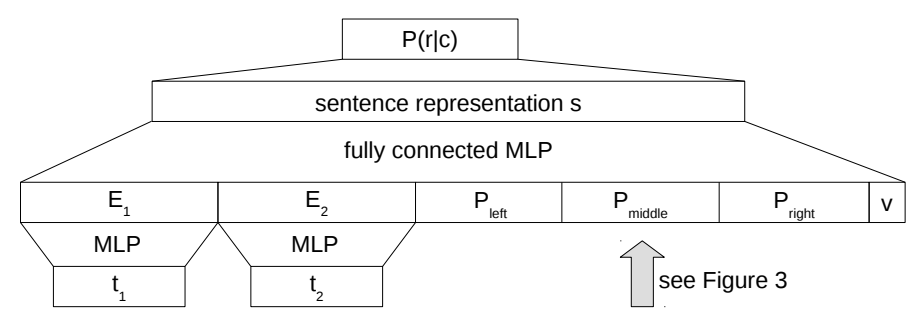

Figure 4: Integration of entity type information into multi-class CNN.

\subsubsection{JoInt TRAINING}

Instead of using prior knowledge about the slots and the expected types of their arguments, we propose to jointly learn entity and relation classification. Following Yaghoobzadeh et al. (2017), we use a convolutional neural network to predict scores for the different types (see Figure 5). We treat this as a multi-label classification task and use the sigmoid function to ensure that the scores for each class are between 0 and 1 . We then use the scores as type vectors $t_{1}$ and $t_{2}$ in Figure 4 . This is similar to the architecture PREDICTED-Hidden from Yaghoobzadeh et al. (2017) with the following differences: We do not use entity embeddings for modeling the relation arguments (see Section 3.1 for our motivation) and also integrate the flag for the order of the relation arguments. This flag is highly relevant for slot filling since there is an inverse slot for almost all slots.

For jointly training the $\mathrm{CNN}$ for entity classification and the CNN for relation classification, we use the following loss function:

$$
L=(1-\alpha) \cdot L_{\mathrm{rel}}+\frac{\alpha}{2} \cdot L_{\text {type1 }}+\frac{\alpha}{2} \cdot L_{\text {type2 }}
$$

The weight $\alpha$ controls the ratio between the relation classification loss $L_{\text {rel }}$ and the losses of entity type classification $L_{\text {type1 }}$ and $L_{\text {type2 }}$. It is tuned on the development set. 


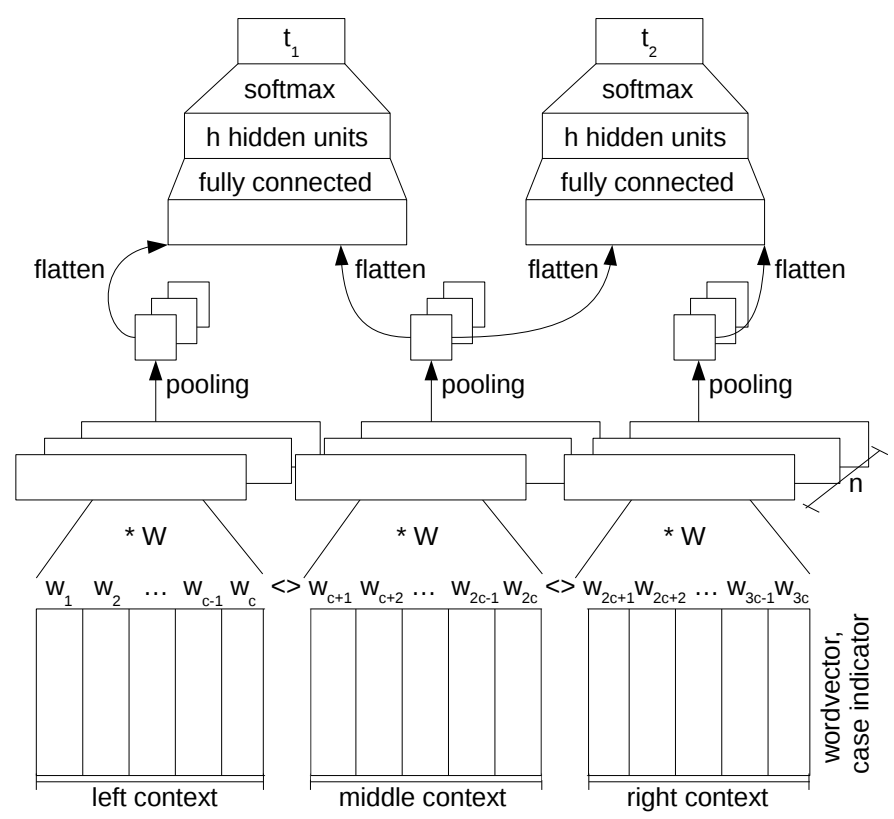

Figure 5: Convolutional neural network for entity type classification; $t_{1}$ and $t_{2}$ are the predictions of the types of the first and second relation argument, respectively.

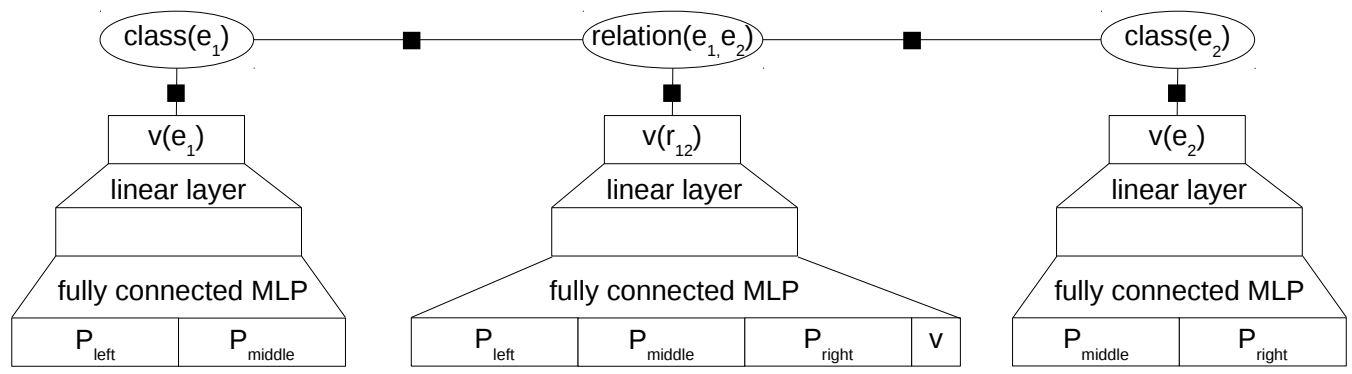

Figure 6: CNN with structured prediction for type-aware slot filling relation classification.

\subsubsection{Structured Prediction}

The third approach for integrating entity information into a convolutional neural network for relation classification is based on structured prediction, as we originally presented for a table filling evaluation of entity and relation recognition (Adel \& Schütze, 2017). While we applied it only to a manually labeled dataset in that previous work, we now adopt it to the slot filling pipeline setting with distantly supervised training data for the first time. 
Figure 6 shows the architecture of the model. Again, the context is split into three parts: left, middle and right context. For each context, a convolutional and 3-max pooling layer computes a representation with weights shared across contexts. For predicting the class of the first entity, we use the representation of the left and middle context; for predicting the class of the second entity, we use the representation of the middle and right context. To calculate scores for the possible relations, all three contexts are used. In contrast to the model we proposed earlier (Adel \& Schütze, 2017), we again use the flag for the order of the relation arguments for classifying the relation. Also, we do not compute representations for entities for slot filling, as motivated in Section 3.1. The scores of the two entity classes and the relation class are then fed into a conditional random field (CRF) output layer which optimizes the following sequence of predictions:

$$
\text { [class of } e_{1} \text {, relation } r_{12} \text { between } e_{1} \text { and } e_{2} \text {, class of } e_{2} \text { ] }
$$

In particular, we apply a linear-chain conditional random field. Thus, the model learns scores for transitions $T$ between the class of the first entity and the relation and between the relation and the class of the second entity. As a result, it approximates the joint probability of entity classes $C_{e_{1}}, C_{e_{2}}$ and relations $R_{e_{1} e_{2}}$ as follows:

$$
P\left(C_{e_{1}}, R_{e_{1} e_{2}}, C_{e_{2}}\right) \approx P\left(C_{e_{1}}\right) \cdot P\left(R_{e_{1} e_{2}} \mid C_{e_{1}}\right) \cdot P\left(C_{e_{2}} \mid R_{e_{1} e_{2}}\right)
$$

Our intuition behind this is that the dependency between relations and entities is stronger than the dependency between the two entities.

Given neural network activations $v$ for the different entity and relation classes, the input sequence $d$ to the CRF layer is

$$
d=\left[v\left(e_{1}\right), v\left(r_{12}\right), v\left(e_{2}\right)\right]
$$

This sequence is padded with a begin and end tag and used to compute the score for a particular sequence $s$ in the following way:

$$
\operatorname{score}(s)=\sum_{i=0}^{n} T_{s_{i} s_{i+1}}+\sum_{i=1}^{n} d_{i s_{i}}
$$

with $T$ being the transition scores (randomly initialized and learned during training) and $d$ storing the neural network activations (see Equation 12). Following Lample et al. (2016), we assume that all variables live in log space and, therefore, use the sum in Equation 13.

For training, we normalize the score of the gold sequence over the scores of all possible sequences. We compute all possible sequences with the forward algorithm. To compute the best path during testing and get probabilities for the different relation classes, we apply the viterbi and forward-backward algorithm, respectively (Rabiner, 1990). 


\section{Experiments and Results}

In this section, we describe our datasets (Section 4.1), and present and discuss our results. We conduct two sets of experiments: First, we evaluate our models in a pure relation classification setup (Section 4.2). Second, we show the performance of the slot filling pipeline when using our models in the slot filler classification component (Section 4.3). Section 4.4 discusses the difference between the results presented in Section 4.2 and Section 4.3. Finally, Section 4.5 sets our results in the context of other state-of-the-art slot filling pipelines.

\subsection{Data}

This subsection describes the different datasets we created for our experiments: Section 4.1.1 presents the training data, Section 4.1.2 reviews the slot filling relation classification benchmarks we use to optimize our models (development set) and to evaluate their performance outside of the slot filling pipeline (test set). Section 4.1.3 describes how we transform the training data which has been created for binary models into a dataset for training multi-class models.

\subsubsection{Training Data}

The slot filling shared task does not offer a training dataset for relation classification models. Therefore, it is necessary to create one. Since manual labeling is expensive and does not scale to large amounts of data, we choose a distantly supervised labeling approach (Mintz et al., 2009). In particular, we create a large set of training examples using distant supervision over Freebase relation instances (Bollacker et al., 2008) and the following corpora:

- TAC source corpus (LDC2013E45)

- NYT corpus (LDC2008T19)

- subset of ClueWeb (Clueweb-URL)

- Wikipedia

- Freebase description fields

Negative examples for each relation are created by extracting sentences with entity pairs with the correct named entity tags for the given slot but without the given relation according to Freebase. However, as mentioned before, Freebase is incomplete. Thus, if a relation between two entities is not stored in Freebase, it does not mean that it does not exist in reality. Therefore, we clean the negative examples with trigger words and patterns: If a trigger/pattern of the given relation appears in the sentence, we do not include it in the set of negative examples. The list of triggers has been compiled manually based on the official 
slot descriptions and examples provided by TAC (Slot-descriptions-URL). It consists of a few high-precision patterns for each slot. By manually investigating random subsets of the filtered examples, we find the pattern set to be very effective in reducing the number of false negative labels.

To reduce the number of false positive labels (and further improve the negative labels), we perform an automatic training data selection process. First, the extracted training samples are divided into $k$ batches. Then, we train one SVM per slot on the annotated slot filling dataset released by Angeli et al. (2014b). Thus, the classifiers are trained on data with presumably correct labels and should, therefore, be able to help in the process of selecting additional data. For each batch of training samples, we use the classifiers to predict labels for the samples and select those samples for which the distantly supervised label corresponds to the predicted label with a high confidence of the classifier. Those samples are, then, added to the training data and the SVMs are retrained to predict the labels for the next batch. This process is depicted in Figure 7.

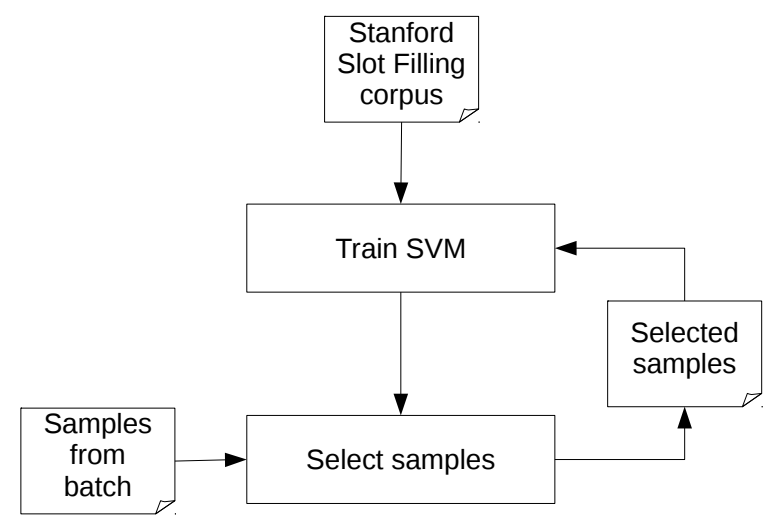

Figure 7: Training data selection process.

\subsubsection{Development Data: Slot Filling Benchmark}

To optimize the parameters and to test the slot filling relation classifiers outside of the slot filling pipeline, we build a slot filling relation classification dataset, leveraging the existing manually labeled system outputs from the previous slot filling evaluations (Adel et al., 2016): We extract the supporting sentences from the system outputs and automatically determine the position of the entity and the filler. Then, we label each sentence as correct or wrong according to the manual assessment provided by the shared task organizers. Due to differences in the offset calculation of some systems, we cannot use all available data: We extract 39,386 relation classification instances out of the 59,755 system output instances which have been annotated as either completely correct or completely incorrect by the shared task organizers. Thus, the resulting dataset has a reasonable number of examples 
with presumably clean labels. For our experiments, we split the data into a development part (data from slot filling evaluations 2012-2013) and a test part (data from slot filling evaluations 2014). We tune the parameters of our models on the development part and use the test part as a first indicator of their performance on unseen data. For more details on the data and a script to reproduce the data, see Adel et al. (2016).

\subsubsection{Data For Multi-Class Models}

Since our training dataset has been created for binary models, the negative examples for each slot need to be processed for the multi-class setting: For example, a negative instance for the slot per:date_of_birth is not automatically a negative instance for the slot per:date_of_death. Therefore, we filter the negative instances in the training data with pattern lists: A negative instance that includes a trigger for any of our positive slots is deleted from the set. The remaining negative instances are labeled with an artificial class N. Finally, we found it beneficial on the slot filling relation classification benchmark to subsample the number of negative instances. Thus, we use the same number of negative instances as non-negative instances.

Note that we only modify the training set and still use the original development and test sets from the slot filling relation classification benchmark for our experiments in order to compare the multi-class models with the binary models.

\subsection{Results on Slot Filling Benchmark}

In this subsection, we present the performance of our models on the slot filling benchmark dataset (which is described in Section 4.1.2). Section 4.2.1 summarizes the baseline models to which we compare our type-aware convolutional neural networks, and Section 4.2.2 describes the evaluation measure. Finally, Section 4.2.3 provides and discusses the performance of our different models.

\subsubsection{Baselines and Support Vector Machines}

In earlier work (Adel et al., 2016), we compared various models on the slot filling relation classification benchmark dataset described in Section 4.1.2. In this paper, we use the same baseline models, namely Mintz++ (Mintz et al., 2009) and MimleRe (Surdeanu et al., 2012). For another comparison, we also train multi-class variants of the SVM models from Adel et al. (2016). For training the multi-class SVM, we apply LinearSVC from scikit learn (LinearSVC-URL) with automatically adjusted class weights and compare the one-vs-rest training strategy (which actually trains binary classifiers by contrasting the examples from one class against the examples from all other classes) with the multi-class training strategy by Crammer and Singer (2001) (which changes the objective function to optimizing multiple classes jointly). The SVM is implemented using liblinear (Fan et al., 2008). 


\subsubsection{Evaluation}

The different models are evaluated using $F_{1}$, the harmonic mean of precision $P$ and recall $R$ of the classifiers:

$$
F_{1}=\frac{2 \cdot P \cdot R}{P+R}
$$

We calculate both slot-wise $F_{1}$ scores and a macro $F_{1}$ score which is the average of the scores over all slots.

\begin{tabular}{|c|c|c|c|c|c|c|c|c|c|c|}
\hline & \multirow[t]{2}{*}{ MinTZ } & \multirow[t]{2}{*}{ MiML } & \multicolumn{3}{|c|}{ SVM } & \multicolumn{5}{|c|}{$\mathrm{CNN}$} \\
\hline & & & binary & O-v-r & $\mathrm{c}-\mathrm{s}$ & binary & multi & $+\mathrm{p}$ & $+\mathrm{j}$ & $+\mathrm{s}$ \\
\hline per:age & .71 & .73 & .74 & .70 & .70 & .76 & .68 & .72 & .66 & .67 \\
\hline per:alternate_names & .03 & .03 & .02 & .00 & .00 & .04 & .00 & .00 & .00 & .00 \\
\hline per:children & .43 & .48 & .68 & .39 & .45 & .61 & .48 & .44 & .44 & .36 \\
\hline per:cause_of_death & .42 & .36 & .32 & .00 & .00 & .52 & .11 & .29 & .00 & .06 \\
\hline per:date_of_birth & .60 & .60 & .67 & .33 & .57 & .77 & .80 & .80 & .73 & .73 \\
\hline per:date_of_death & .45 & .45 & .54 & .60 & .62 & .48 & .51 & .46 & .39 & .59 \\
\hline per:empl_memb_of & .36 & .37 & .36 & .21 & .15 & .37 & .28 & .29 & .25 & .28 \\
\hline per:location_of_birth & .22 & .22 & .27 & .31 & .33 & .23 & .36 & .20 & .34 & .35 \\
\hline per:loc_of_death & .41 & .43 & .34 & .32 & .35 & .28 & .28 & .19 & .25 & .21 \\
\hline per:loc_of_residence & .11 & .18 & .33 & .11 & .08 & .23 & .15 & .06 & .22 & .25 \\
\hline per:origin & .48 & .46 & .64 & .04 & .02 & .39 & .11 & .30 & .13 & .17 \\
\hline per:schools_att & .78 & .75 & .71 & .58 & .62 & .55 & .45 & .47 & .56 & .68 \\
\hline per:siblings & .59 & .59 & .68 & .71 & .68 & .70 & .73 & .54 & .63 & .68 \\
\hline per:spouse & .23 & .27 & .32 & .45 & .49 & .30 & .39 & .49 & .36 & .30 \\
\hline per:title & .39 & .40 & .48 & .51 & .45 & .46 & .42 & .43 & .44 & .48 \\
\hline org:alternate_names & .46 & .48 & .62 & .59 & .52 & .66 & .58 & .55 & .50 & .58 \\
\hline org:date_founded & .71 & .73 & .70 & .60 & .60 & .71 & .63 & .65 & .74 & .69 \\
\hline org:founded_by & .62 & .65 & .74 & .70 & .68 & .68 & .71 & .43 & .74 & .73 \\
\hline org:loc_of_headqu & .19 & .20 & .42 & .14 & 11 & .45 & .24 & .42 & .21 & .34 \\
\hline org:members & .06 & .16 & .13 & .15 & .31 & .04 & .17 & .07 & .17 & .11 \\
\hline org:parents & .14 & .17 & .20 & .10 & .11 & .16 & .14 & .09 & .12 & .10 \\
\hline org:top_memb_empl & .44 & .46 & .55 & .54 & .51 & .53 & .55 & .49 & .58 & .58 \\
\hline macro $F_{1}$ & .40 & .42 & .48 & .37 & .38 & .45 & .40 & .38 & .38 & .41 \\
\hline
\end{tabular}

Table 1: $F_{1}$ results on slot filling benchmark test data (from 2014). The columns show our binary and multi-class SVMs and CNNs as well as our type-aware CNN models in comparison to two standard baseline models for relation classification: Mintz and Miml (see Section 4.2.1 for more information). o-v-r: one-vs-rest; c-s: Crammer and Singer; p: pipeline (Section 3.3.1), j: joint training (Section 3.3.2), s: structured prediction (Section 3.3.3). 
ADEL \& SCHÜTZE

\subsubsection{Results}

Table 1 provides slot-wise results for the baseline models, the SVMs as well as for the different CNN setups: binary CNNs, a multi-class CNN without entity type information, a multi-class CNN with slot-based entity types $(+\mathrm{p})$, a multi-class $\mathrm{CNN}$ with entity type probabilities jointly trained with the relation classification $\mathrm{CNN}(+\mathrm{j})$, and a multi-class CNN with a structured prediction (CRF) output layer which optimizes a sequence of entity and relation classes $(+\mathrm{s})$. Note that Table 1 shows results for 22 slot types instead of 65 slot types mentioned in Section 2.1. The reason is that we merged all location slots and all inverse slot pairs into one slot type (see Section 3.1). For example, our slot type per:children actually covers both original slot types per:children and per:parents, and our slot type org:founded_by covers even four slot types, namely org:founded_by, per:organizations_founded, org:organizations_founded and gpe:organizations_founded. As a result, our merged slot types in Table 1 actually cover 54 out of all 65 slot types. We provide an overview which original slot types we cover with our models in Table 7 in the appendix. For the remaining slot types, we were not able to extract enough training data with distant supervision to train machine learning models. For those slots, our slot filling pipeline falls back to pattern matching.

In general, the binary models perform better than the multi-class models, even when adding entity type information to the latter. For example, the binary CNN achieves better results than the multi-class CNN without entity type information for 13 out of 22 slot types and better results than any multi-class CNN (with or without entity type information) for nine out of 22 slot types. We assume that one reason might be that the convolutional filters of the binary models can concentrate on learning features for just one relation type while the filters of the multi-class models need to learn features which can be used to discriminate all relation types. All binary models outperform the baseline models MiNTZ and MimL. The binary SVM performs slightly better than the binary CNN. In contrast to these results, the multi-class CNN outperforms the multi-class SVM. However, both models seem to struggle with the large number of output classes.

While the model with jointly trained entity classification achieves slightly better results on the development set than the multi-class model without named entity information (0.53 vs. 0.52$)$, this improvement is not transferred to the test set. The structured prediction model generalizes better to an unseen test set than the other type-aware models and comes closer to the results of the binary CNN. Slots for which entity type information seems to help the most are per:date_of_birth and per:location_of_birth, two slots with similar contexts. Similarly, type-aware CNNs achieve the best results for org:date_founded and org:founded_by. Despite their lower results on average, the multiclass models generalize better to unseen test data than the binary models for some slots, such as per:date_of_death, per:schools_attended or per:spouse.

In the next section, we provide results for using the different models in the slot filling pipeline. 


\subsection{Slot Filling Pipeline Results}

In this subsection, we present the performance of our slot filling pipeline (described in detail in Section 2.2) on the official slot filling evaluation data from 2015. It consists of 1951 queries with manual assessments of the outputs of the systems that were submitted to the shared task evaluations. 930 of those queries are multi-hop queries, i.e., they require the system to first fill one slot and then fill a second slot based on the answers of the first slot (see Section 2.1). Section 4.3.1 presents the different system configurations we evaluate. Section 4.3.2 describes the evaluation measures and Section 4.3.3 provides and discusses the results.

\subsubsection{System Configurations}

In this experiment, we run the whole slot filling pipeline as described in Section 2.2. The different configurations we evaluate differ from one another in terms of the relation classification models that are used in the slot filler classification component. Note that all configurations use the pattern matching module in addition to the machine learning models. The numbers we provide in the following to distinguish the different configurations correspond to the numbers in Table 2. System I only uses binary SVMs in combination with patterns. Thus, it can be used to assess the impact of CNNs in general. System II combines binary SVMs and binary CNNs. To assess the impact of joint training with entity types also for the binary models, we add another configuration, system III, that uses binary SVMs and binary CNN models which are jointly trained on entity typing and relation classification. Thus, in contrast to system II, it has the ability of correcting wrong entity types from the candidate extraction component of the pipeline. System IV uses multi-class SVMs instead of binary SVMs as in system I. For the multi-class SVMs, we use the one-vs-rest training strategy since this led to slightly better results on the development part of the slot filling relation classification benchmark (0.51 vs. 0.50). System V combines multi-class SVMs with multi-class CNNs. Thus, comparing system II to system V allows us to assess the performance difference of binary and multi-class models for slot filling. Finally, systems VI, VII and VIII combine multi-class SVMs with type-aware multi-class CNNs, our main contribution in this paper. System VI integrates pipeline-based CNNs, as described in Section 3.3.1, into the slot filling pipeline. In system VII, we apply CNNs that have been jointly trained on entity and relation classification, as presented in Section 3.3.2. The last configuration, system VIII, uses CNN models trained with structured prediction of both entity and relation classes. Note that we only compare pure binary to pure multiclass classification modules in this paper. We also experimented with combining binary SVMs with multi-class CNNs but did not obtain additional performance gains with this setup. 
ADEL \& SCHÜTZE

\subsubsection{Evaluation}

The slot filling pipeline is evaluated using the official measures from the shared task. In particular, we report two measures, one based on the micro $F_{1}$ score (the $F_{1}$ score over all examples, giving slots with more examples higher weight), and another one based on the macro $F_{1}$ score (the average of slot-wise $F_{1}$ scores, weighting all slots equally): "CSLDC max micro" is the micro precision, recall and $F_{1}$ score over all queries (thus, "micro"). If the hop 0 sub-query occurred several times in the query set (with different hop 1 subqueries), that answer to the hop 0 sub-query is scored which leads to the maximum results over both hops (thus, "max"). "CSLDC max macro" is the average $F_{1}$ score over all slots (thus, "macro"). All scores in this section are calculated using the official shared task scoring scripts. Their readme file provides more details on the scoring procedure (Cold-start-scoring-scripts-URL).

\subsubsection{Results}

Table 2 provides the scores of our slot filling system with our newly introduced classification models in comparison to SVMs and CNNs (in both binary and multi-class variants) without entity type information.

In contrast to the slot filling relation classification results (see Table 1), most of the multi-class models (systems IV-VIII in Table 2) perform comparable or even better than the binary models (systems I-III). The best micro $F_{1}$ results for hop 0 and all (both hops) are achieved by using multi-class classification models (systems V,VII). The multi-class models have higher precision than the binary models across all hops (hop 0, hop 1 and all). The multi-class models with joint training of entity types and relation classification (system VII) achieves the highest overall micro $F_{1}$ score. In terms of macro $F_{1}$, the binary model with jointly learned types (system III) and the multi-class model with structured prediction (system VIII) perform best. This suggests that the opportunity to correct wrong entity types from the candidate extraction component is crucial and that joint modeling with entity types especially improves the performance on rare relations.

\subsection{Discussion of Results: Benchmark vs. Pipeline}

The performance ranking of the models on the benchmark dataset for slot filling relation classification (Table 1) is different to the performance ranking in the slot filling pipeline evaluation (Table 2). This seems to be in contradiction to the positive correlation of results we reported earlier (Adel et al., 2016). We assume that the reason is domain mismatch. While the positive correlation was calculated for running the pipeline on 2013/2014 slot filling evaluation data (Adel et al., 2016), the pipeline is now run on 2015 evaluation data. In 2015, the evaluation corpus for slot filling was changed, introducing many more discussion forum documents and significantly changing the ratio of domains (see Table 3 ). This leads to a severe domain mismatch challenge for the components of the slot filling pipeline 
Type-aware Convolutional Neural Networks for Slot Filling

\begin{tabular}{|c|c|c|c|c|c|c|c|}
\hline & & & & & micro & & macro \\
\hline & & SVM & $\mathrm{CNN}$ & $P$ & $R$ & $F_{1}$ & $F_{1}$ \\
\hline & (I) & binary & - & 29.12 & 26.18 & 27.57 & 32.50 \\
\hline & (II) & binary & binary & 31.79 & 28.23 & 29.91 & 34.20 \\
\hline & (III) & binary & binary $+\mathrm{j}$ & 30.11 & 28.23 & 29.14 & 34.81 \\
\hline $\begin{array}{l}0 \\
0\end{array}$ & (IV) & multi & - & 25.25 & 12.07 & 16.33 & 18.44 \\
\hline 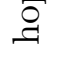 & $(\mathrm{V})$ & multi & multi & 34.42 & 26.66 & 30.04 & 32.82 \\
\hline & (VI) & multi & multi $+\mathrm{p}$ & 23.58 & 28.55 & 25.83 & 30.91 \\
\hline & (VII) & multi & multi $+\mathrm{j}$ & 32.42 & 27.84 & 29.95 & 33.14 \\
\hline & (VIII) & multi & multi $+\mathrm{s}$ & 33.33 & 27.68 & 30.25 & 33.98 \\
\hline & (I) & binary & - & 7.36 & 4.78 & 5.80 & 6.13 \\
\hline & (II) & binary & binary & 9.80 & 7.00 & 8.17 & 8.28 \\
\hline & (III) & binary & binary $+\mathrm{j}$ & 8.90 & 6.56 & 7.55 & 8.73 \\
\hline & (IV) & multi & - & 7.11 & 3.67 & 4.84 & 4.34 \\
\hline 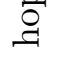 & $(\mathrm{V})$ & multi & multi & 12.59 & 3.89 & 5.94 & 7.78 \\
\hline & (VI) & multi & multi $+\mathrm{p}$ & 6.62 & 3.00 & 4.13 & 4.66 \\
\hline & (VII) & multi & multi $+\mathrm{j}$ & 13.47 & 5.00 & 7.29 & 8.15 \\
\hline & (VIII) & multi & multi $+\mathrm{s}$ & 12.24 & 5.22 & 7.32 & 9.24 \\
\hline & (I) & binary & - & 21.75 & 17.30 & 19.27 & 23.06 \\
\hline & (II) & binary & binary & 23.80 & 19.42 & 21.39 & 24.92 \\
\hline & (III) & binary & binary $+\mathrm{j}$ & 22.52 & 19.23 & 20.75 & 25.47 \\
\hline$=$ & (IV) & multi & - & 17.38 & 8.58 & 11.49 & 13.39 \\
\hline$\approx$ & $(\mathrm{V})$ & multi & multi & 29.60 & 17.20 & 21.76 & 23.86 \\
\hline & (VI) & multi & multi $+\mathrm{p}$ & 20.02 & 17.94 & 18.92 & 21.51 \\
\hline & (VII) & multi & multi $+\mathrm{j}$ & 27.97 & 18.36 & 22.17 & 24.20 \\
\hline & (VIII) & multi & multi $+\mathrm{s}$ & 27.70 & 18.36 & 22.08 & 25.12 \\
\hline
\end{tabular}

Table 2: Slot filling pipeline results for our different models and model combinations. Evaluation measure: CSLDC $\max$ micro/macro. p: pipeline, $\mathrm{j}$ : joint training, s: structured prediction as in Table 1.

and reduces the correlation with the benchmark dataset which has been built based on 2012-2014 data. Nevertheless, participants of the slot filling shared task only have previous evaluation data available for developing and tuning their models. Therefore, we argue that it is still important to also evaluate models on the slot filling relation classification benchmark. 
ADEL \& SCHÜTZE

\begin{tabular}{l|rr|rr} 
& \multicolumn{2}{|c|}{ until 2014} & \multicolumn{2}{c}{2015} \\
& number & ratio & number & ratio \\
\hline news documents & $1,000,257$ & $47.65 \%$ & 8,938 & $18.19 \%$ \\
web documents & 999,999 & $47.63 \%$ & 0 & $0 \%$ \\
discussion forum & 99,063 & $4.19 \%$ & 40,186 & $81.81 \%$
\end{tabular}

Table 3: Domains in slot filling corpora.

\subsection{Comparison with State of the Art}

Finally, we set our results in the context of state of the art on the 2015 evaluation dataset for slot filling.

\begin{tabular}{l|l|r|c} 
rank & team & micro $F_{1}$ & distant supervision? \\
\hline 1 & Stanford & 31.06 & no \\
\hline 2 & UGhent & 22.38 & yes \\
& CIS (with type-aware CNN) & 22.17 & yes \\
3 & CIS (our official submission) & 21.21 & yes \\
4 & UMass & 17.20 & yes \\
5 & UWashington & 16.44 & yes \\
\hline median & - & 15.32 & -
\end{tabular}

Table 4: State-of-the-art results for slot filling. Stanford: (Angeli et al., 2015), UGhent: (Sterckx et al., 2015), CIS: (Adel \& Schütze, 2015), UMass: (Roth et al., 2015), UWashington: (Soderland et al., 2015). The third line ("CIS with type-aware CNN") is our best result from Table 2. Our official submission (forth line) did not include type-aware models.

Table 4 shows that our official submission to the shared task in 2015, which did not include type-aware models, was ranked at third position. With our type-aware convolutional neural networks, we can improve our result. Our pipeline performs comparable to the second rank now. It is considerably better than the system on rank 4 and performs clearly above median. Only the results of the top-performing system are still superior. As also described in Section 6, there are two differences relevant to this: First, the top-performing system does not use information retrieval, like our system and most other systems, but stores preprocessed versions of the corpus in a database, including an index for all occurring entities. This requires extensive corpus preprocessing (such as the identification of all entities along with their positions in the documents) and data storage (the source corpus contains millions of documents) but makes it possible to access the query entities directly at test time. However, this approach is only possible with prior access to the whole corpus and cannot be applied to changing environments. In contrast, our retrieval-based pipeline 
is more flexible since it processes only those documents relevant to the input query. The second, and arguably more important, difference in terms of final performance is that the training dataset of the top-performing system has been labeled manually via crowdsourcing. In contrast, the datasets of the other systems are created with distant supervision. When training our models, we observed large performance differences depending on the quality of the training data. Therefore, we suspect that the main reason for the superior performance of the system by Angeli et al. (2015) is their training data. Unfortunately, obtaining manual labels is time-consuming and challenging, even in the context of crowdsourcing. An example is the extension of the knowledge base schema to new relations or a more fine-grained distinction of existing relations which would always require manual relabeling. Therefore, automatic methods like distant supervision are still of high relevance. Among the systems using automatically created training data, our pipeline is state of the art.

\section{Analysis}

In this section, we analyze the behavior of the slot filling system in more detail in order to see which pipeline components need to be improved in the future. Section 5.1 analyzes the recall of the different components of the slot filling pipeline, showing which components are responsible for which recall loss. Section 5.2 presents a manual analysis of wrong system outputs, categorizing the errors with respect to which pipeline component is responsible for them. Finally, Section 5.3 provides several ablation studies, indicating the impact of entity linking, coreference resolution and type-aware neural networks.

\subsection{Recall Analysis}

Our first analysis investigates the recall of the different components and is similar to the analysis by Pink et al. (2014). In particular, we evaluate the components of our system before the slot filler classification module. Thus, we measure which recall our system could achieve with a perfect slot filler classification module that does not lose any recall.

Figure 8 shows the results on the slot filling assessment data from 2015 for hop 0: Information retrieval with TERRIER IR and fuzzy string match is able to achieve a recall of $78.82 \%$. The entity linking component hurts recall a bit. However, it also increases precision which leads to better overall results (cf., Section 5.3.1). Evaluating only the top 100 documents instead of all extracted documents from TERRIER (maximum 300), leads to a recall loss of almost $5 \%$. Thus, allowing the slot filling system a longer run time for processing all extracted documents could lead to a higher final recall (but potentially also to more false positive extractions and, thus, a lower precision). As mentioned before, choosing only the 100 most relevant documents has led to the best time-performance tradeoff on data from previous evaluations (2013 and 2014). The sentence extraction component extracts the relevant sentences quite successfully with an additional recall loss of only 


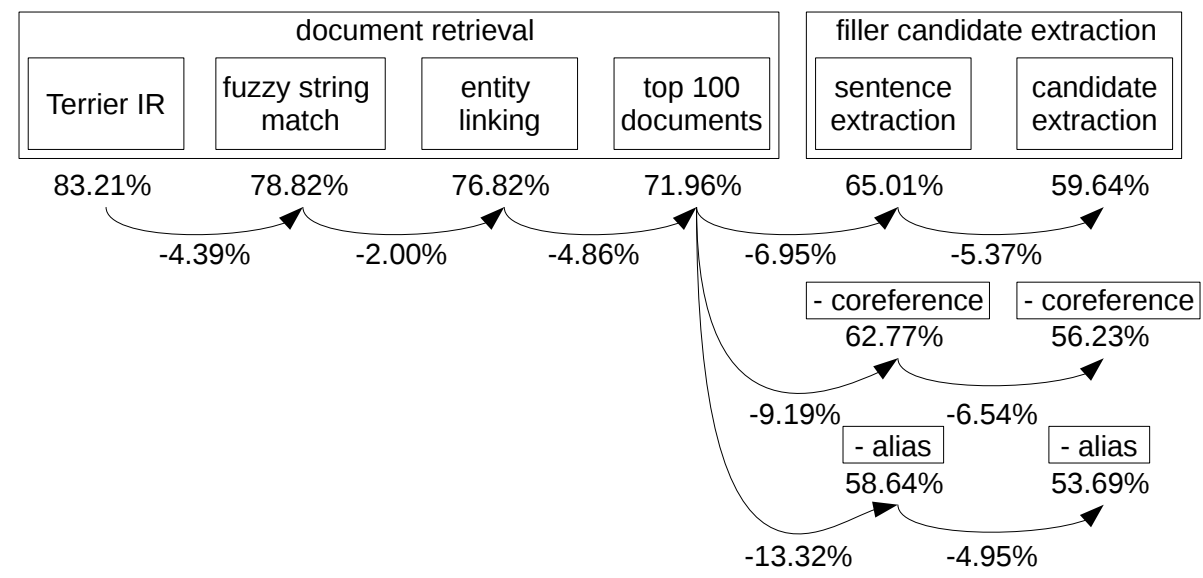

Figure 8: Analysis of recall after the application of the different pipeline components.

6.95\%. Evaluating this component in more detail shows the importance of coreference resolution and aliases: The recall loss without coreference resolution is almost $10 \%$, the recall loss without aliases is more than $13 \%$. Finally, the candidate extraction component is able to extract most of the relevant candidates, yielding an overall recall of $59.64 \%$ before slot filling relation classification. Without coreference resolution for sentence extraction, the overall recall is $56.23 \%$, without alias information for sentence extraction, the overall recall is $53.69 \%$. Assuming a perfect slot filler classification component with $P=100 \%$ and $R=100 \%$, the maximum $F_{1}$ score of the whole slot filling system would be $74.72 \%$. This number is about twice as high as the performance of the best slot filling system 2015 (Angeli et al., 2015) (see Table 4) but still low compared to other NLP tasks. This illustrates the difficulties of the slot filling task and the importance of all individual components of the pipeline since especially recall losses cannot be recovered by subsequent components.

\subsection{Error Analysis}

In our second analysis, we manually analyze 120 errors of our system from the official 2015 evaluations, i.e., its wrong (false positive) predictions. While Section 5.1 shows the recall loss of the different pipeline components, this analysis categorizes which component is responsible for which false positive prediction, and as a result, for a precision loss of the pipeline. Table 5 shows which pipeline component is responsible for how many errors. The numbers do not sum to 1 since for $7 \%$ of the cases, we could not unambiguously identify a single component as the error source.

The alias component especially struggles with acronyms which can refer to several entities. An example is NL which is an acronym for "National League" in the document collection but got wrongly recognized as an acronym for the query entity "Nest Labs". In the candidate extraction component, most errors ( $16 \%$ of $21 \%$ ) occur in the named entity 
Type-aware Convolutional Neural Networks for Slot Filling

\begin{tabular}{lr} 
Error category & ratio \\
\hline Alias component & $9 \%$ \\
Entity linking component & $2 \%$ \\
Candidate extraction component & $21 \%$ \\
Classification component & $61 \%$
\end{tabular}

Table 5: Error analysis of the pipeline.

recognition part. For example, "Bloomberg" is wrongly tagged as organization although it is a person in the given context ("... people like Bloomberg ..."). Similarly, "Heinz" gets tagged as person although it is an organization in the given context ("MacDonald's dropping Heinz after CEO change"). Nested named entities are also a challenge: In "Tom Clancy games", for example, "Tom Clancy" gets tagged as a person although the whole phrase actually forms a single entity. For some instances (4\% of $21 \%$ ), the document has been incorrectly split into sentences and in the remaining cases (1\% of $21 \%$ ), coreference resolution failed. The classification component faces a very challenging task since most extracted filler candidates are false positives. Thus, it has to establish precision while keeping as much recall as possible. Based on a manual inspection of errors, the most important challenge for the classification component is long contexts which mention several relations between several entity pairs. An example is "Mikhail Kalashnikov, designer of the famed Russian AK-47 assault rifle, died on Monday in his home city of Izhevsk, an industrial town 1,300 km east of Moscow, local media reported" from which the relation per:location_of_death between Mikhail Kalashnikov and Moscow is extracted. Thus, our classification component correctly recognizes the relation trigger "died [...] in" but assigned it to the wrong relation arguments. This finding is in line with the study by Huang et al. (2017) who also identified long context as one of the main challenges in slot filling relation classification.

\subsection{Ablation Studies}

In our last analysis, we present ablation studies showing the impact of entity linking, coreference resolution and type-aware neural networks. We focus on entity linking and coreference resolution since the design choices of whether or not to integrate them into a slot filling system are among those with the highest disagreement among slot filling researchers (see our description of related work in Section 6). Furthermore, the type-aware neural networks for slot filling are the main contribution of this paper. Table 6 compares the performance of our slot filling system with and without entity linking, coreference resolution, and type-aware neural networks, respectively. 
ADEL \& SCHÜTZE

\begin{tabular}{|c|c|c|c|c|c|}
\hline & & $P$ & $R$ & $F_{1}$ & $\Delta F_{1}$ \\
\hline \multirow{4}{*}{ 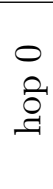 } & joint & 32.42 & 27.84 & 29.95 & \\
\hline & without entity linking & 31.30 & 27.92 & 29.51 & -0.44 \\
\hline & without coreference & 32.13 & 25.87 & 28.66 & -1.29 \\
\hline & without CNN & 25.25 & 12.07 & 16.33 & -13.62 \\
\hline \multirow{4}{*}{ ڤ્a } & joint & 13.47 & 5.00 & 7.29 & \\
\hline & without entity linking & 11.81 & 4.78 & 6.80 & -0.49 \\
\hline & without coreference & 11.80 & 4.22 & 6.22 & -1.07 \\
\hline & without CNN & 7.11 & 3.67 & 4.84 & -2.45 \\
\hline \multirow{4}{*}{ テี } & joint & 27.97 & 18.36 & 22.17 & \\
\hline & without entity linking & 26.56 & 18.31 & 21.68 & -0.49 \\
\hline & without coreference & 27.25 & 16.88 & 20.85 & -1.32 \\
\hline & without CNN & 17.38 & 8.58 & 11.49 & -10.68 \\
\hline
\end{tabular}

Table 6: Impact of entity linking and coreference resolution and type-aware convolutional neural networks on the slot filling pipeline.

\subsubsection{IMPACT OF ENTITY LINKING}

The system performance is slightly reduced by omitting entity linking. However, the difference of the $F_{1}$ scores is rather small. This shows that the main challenges of the system lie in other components and ambiguous names play a rather small role for the final results of the system.

\subsubsection{Impact of COREFEREnCE}

The $F_{1}$ score drops by 1.3 points when omitting coreference resolution. As expected, the impact on recall is higher than the impact on precision. However, also precision is reduced. This is because the number of true positives is reduced considerably (from 398 to 366) when the system does not use coreference information. The number of false positives is also lower, but the final results show that the impact of the number of true positives is larger.

\subsubsection{Impact of Neural Networks}

Type-aware CNNs have the largest impact on performance. They improve both precision and recall considerably. In contrast to SVMs, the usage of word embeddings allows the CNNs to detect synonyms or phrases which are similar but not the same as the ones learned during training. Training them jointly with entity classification allows them to benefit from the mutual dependencies between entity and relation classes. 


\subsection{Discussion: Lessons Learned}

When developing the slot filling system and training the relation classification models, we had to solve several challenges. In this section, we report on lessons we have learned when developing the slot filling pipeline. Those are mainly qualitative statements which we have found by manually inspecting intermediate system outputs and results in the development process. Thus, many of them are based on prior experiments and not on concrete numbers reported in the paper.

For the slot filling pipeline, it is especially useful to keep the extracted filler candidates and contexts as clean as possible, e.g., by applying genre-specific document processing steps and manually cleaning filler lists for string slots. Moreover, it is important for the recall of the system to extend the integration of coreference resolution, for instance, by applying it to both relation arguments. Our recall analysis and ablation study also show the positive impact of coreference resolution on the overall results. Based on our error analysis, the candidate extraction module and the classification module are responsible for most of the errors of the overall system. Thus, for future work, it is essential to focus on those two components.

The performance of the relation classification models is mainly influenced by their training data and the input they get from the pipeline. If the input is very long, the models might extract wrong relations or relations between different entities than the query entity and the filler candidate. This is a particular challenge of slot filling since the quality of the inputs to the relation classification models directly depends on the previous system components. The significant difference of the top-ranked slot filling system to all the other systems in 2015 emphasizes the importance of a high-quality training dataset for slot filling relation classification. In an analysis of random subsamples of our training dataset before and after reducing wrong labels with patterns and self-training, we saw the importance of cleaning the noisy labels from distant supervision. Despite our automatic cleaning steps, our dataset still includes noise and a promising future research direction might be the exploration of techniques for further enhancing the data or collecting new data without distant supervision. Furthermore, our results suggest that multi-class models with entity type information are a promising direction for future research on slot filling.

\section{Related Work}

The slot filling shared task has been held since 2009. There are about 20 teams participating each year. Most systems apply a modular pipeline structure and combine multiple approaches, such as distant supervision and patterns (Surdeanu \& Ji, 2014). In 2015, we were one of the first teams using neural networks (Adel \& Schütze, 2015; Angeli et al., 2015; Roth et al., 2015).

In this section, different approaches for implementing the slot filling pipeline are de-

scribed, followed by a more detailed description of two systems that are most relevant to 
our work: the top-ranked system from 2013 (Roth et al., 2013) since we use their distantly supervised patterns and similar features in our support vector machines; and the winning system from 2015 (Angeli et al., 2015) since we evaluate our system on the assessment data from 2015. Finally, we summarize more recent developments in slot filling research.

Most slot filling systems consist of an information retrieval-based pipeline of different modules. Exceptions are, for example, the systems by Wazalwar et al. (2014) and Angeli et al. (2015), which rely on relational databases consisting of one table storing all sentences from the corpus and another table storing all entity mentions. Most groups expand the query with aliases (i.a., Roth et al., 2013; Angeli et al., 2013; Nguyen et al., 2014; Hong et al., 2014; Bao et al., 2016; Chang et al., 2016). Our system follows this line of work and uses information retrieval and query expansion to extract relevant documents and cover alternate names and spelling variations.

For sentence extraction, only a subset of systems use coreference information (e.g., Nguyen et al., 2014; Qin et al., 2015; Angeli et al., 2015; Yang et al., 2016; Yu et al., 2016; Zhang et al., 2016). In all our experiments and analysis, coreference information improves the final results though. Pink et al. (2014) mention the long computing time of coreference resolution systems as a major drawback. This is why we make our coreference resource publicly available. Together with our positive results, it can help convince other researchers to integrate this component which we consider very important, especially for the recall of a slot filling system. Even fewer systems apply entity linking or another form of disambiguating different entities with the same name (e.g., Gonzàlez et al., 2012; Angeli et al., 2015). Our results with entity linking are mixed: Although it slightly improves the final pipeline results, it leads to recall losses due to wrong links.

Especially in 2012 and 2013, many systems relied only on pattern matching for identifying slot fillers (i.a., Li et al., 2012; Gonzàlez et al., 2012; Byrne et al., 2013). Now, more and more teams use machine learning models for slot filling relation classification, such as naive Bayes (Byrne, Fenlon, \& Dunnion, 2014), logistic regression (Nguyen et al., 2014; Angeli et al., 2015; Yang et al., 2016; Zhang et al., 2016), conditional random fields (e.g., Zhang et al., 2014; Kisiel et al., 2015) or support vector machines (e.g., Roth et al., 2013, 2015; Zhang et al., 2016). Mintz and MimL, our baseline systems in Section 4.2, are used by, i.a., Nguyen et al. (2014), Angeli et al. (2014a) and Zhang et al. (2016). More recently, participants also train neural networks, such as bidirectional gated recurrent units (Bao et al., 2016), bidirectional long short-term memory (LSTM) networks (Qin et al., 2015; Angeli et al., 2015; Yang et al., 2016; Yu et al., 2016; Zhang et al., 2016; Chang et al., 2016) or convolutional neural networks (Feys et al., 2014; Roth et al., 2015; Bao et al., 2016; Zhang et al., 2016). In 2015, we were one of the first to show the success of neural networks, especially convolutional neural networks, on this task which has led to their increasing popularity.

With the exception of, e.g., Feys et al. (2014), Nguyen et al. (2014), Angeli et al. (2015), Yang et al. (2016) or Zhang et al. (2016) who use human labels or manual cleaning of noisy labels, e.g., in connection with active learning, the machine-learning models are 
trained with distant supervision. In this work, we approach the problem of noisy labels by an automatic self-training procedure. A subset of the data we use for self-training includes crowdsourced annotations but the actual cleaning process is fully automatic and uses machine learning methods. Thus, it scales better to larger datasets than manual cleaning or manual labeling. While some of the participants use binary models, i.a., Roth et al. $(2013,2015)$ or Bao et al. (2016), others train multi-class models, i.a., Feys et al. (2014), Angeli et al. (2015), Roth et al. (2015) and Yang et al. (2016). In this study, we provide a direct comparison of the performance of binary and multi-class models.

Yang et al. (2016) formulate entity type constraints and use integer linear programming (ILP) to combine them with relation classification. In contrast, our type-aware models are trained end-to-end and do not rely on hand-crafted hard constraints. Instead, they are able to learn correlations between entity and relation classes from data. In the relation extraction community, the joint modeling of entity types and relations is known to improve results (cf., Roth \& Yih, 2007; Yao, Riedel, \& McCallum, 2010; Miwa \& Sasaki, 2014). However, only very few approaches use neural models for joint modeling as we do in this research. Examples are Miwa and Bansal (2016) or Pawar, Bhattacharyya, and Palshikar (2017) but both of them apply their models to clean datasets which have been manually labeled with entity types and relations. In contrast, we conduct our experiments on distantly supervised slot filling which provides neither clean labels for entity nor for relation types. We show that it is possible to use joint training of entity and relation classification in order to reduce the problem of error propagation in the slot filling pipeline. The positive results of our type-aware multi-class CNNs may motivate other researchers in slot filling or general relation extraction to extend their neural models with entity type information or other features which are known to be useful for relation classification with traditional models.

The top-ranked system in 2013 (Roth et al., 2013) follows the main trends in slot filling and applies a modular system based on distant supervision which is called RELATIONFACTORY. Its pipeline is similar to ours except that it uses neither entity linking nor coreference resolution. Following Roth et al. (2013), we use their distantly supervised patterns and add skip n-gram features to the feature set of our support vector machines. An important difference of our models, however, is that we also integrate neural networks and train not only binary models but also multi-class models with and without entity type information.

The top-performing system in 2015 (Angeli et al., 2015) uses manually labeled training data (Angeli et al., 2014b) as well as a bootstrapped self-training strategy in order to avoid distant supervision. In contrast to most other slot filling systems, they do not apply a pipeline system based on information retrieval but store preprocessed versions of all sentences and entity mentions from the source corpus in a relational database which they access during evaluation. As relation extractors, they apply a combination of patterns, an open information extraction system, logistic regression, a bidirectional long short-term memory network and special extractors for website and alternate names slots. In contrast to their system, we apply a traditional slot filling pipeline based on information retrieval 
and train convolutional neural networks. In earlier work (Adel \& Schütze, 2015), we also combined convolutional and recurrent neural networks and found that adding recurrent neural networks increased the performance only slightly. Thus, we assume that the main reason for their better performance is the less noise in the labels of their training data.

Last, we summarize more recent developments in slot filling research: Yu and Ji (2016) present a method based on trigger extraction from dependency trees which does not require (distantly) supervised labels and can work for any language as long as named entity recognition, part-of-speech tagging, dependency parsing and trigger gazetteers are available. Huang et al. (2017) follow our work in extracting training and development data and in using convolutional neural networks for slot filling relation classification. They input dependency paths into the network and apply attention in order to account for the larger middle contexts in slot filling relation classification. In contrast to their work, we extend the convolutional neural network in this paper to not only doing relation classification but jointly learning to classify entities and relations. Recently, Zhang et al. (2017) propose position-aware attention which calculates attention weights based on the current hidden state of their LSTM, the output state of the LSTM and the position embeddings which encode the distance of the current word to the two relation arguments. Moreover, they publish a supervised relation extraction dataset, obtained by crowdsourcing, for training slot filling relation classification models. Chaganty et al. (2017) address the issue of evaluating new slot filling systems outside of the official shared task evaluations. They build an evaluation method based on importance-sampling and crowdsourcing which they make publicly available.

\section{Conclusion}

In this paper, we proposed different type-aware convolutional neural network architectures for slot filling. After describing our slot filling pipeline, we focused on its relation classification component. Slot filling relation classification is a task with a particularly noisy setup due to distantly supervised data at training time and error propagation through the pipeline at test time. We were one of the first groups to show that convolutional neural networks are successful classification models in this scenario. We proposed three techniques of incorporating entity type information: a pipeline-based, a joint training and a structured prediction approach. In our experiments, we compared binary and multi-class models and showed that the multi-class models improved the final performance of the slot filling pipeline. The model trained jointly on entity and relation classification achieved the best micro $F_{1}$ scores while the model with structured prediction performed best in terms of macro $F_{1}$. Finally, we presented several analyses to assess the impact and errors of the different components of the pipeline, a very important aspect which is not evaluated in the official slot filling shared task. Our recall analysis showed the importance of aliases and coreference resolution. Our manual error analysis revealed that the candidate extraction component (especially the named-entity-recognition module) and the classification 
component are responsible for most of the errors of the system. Finally, ablation studies confirmed the large positive impact of our type-aware convolutional neural network on the performance of the whole slot filling pipeline.

We publish our complete slot filling system, including the source code and the presented models (http://cistern.cis.lmu.de/CIS_SlotFilling), as well as our coreference resource (http://cistern.cis.lmu.de/corefresources) along with this paper.

\section{Acknowledgments}

Heike Adel is a recipient of the Google European Doctoral Fellowship in Natural Language Processing and this research is supported by this fellowship. This work is also supported by the European Research Council (ERC \#740516) and by DFG (grant SCHU 2246/4-2).

\section{Appendix A. Overview of Slots}

As described in Section 3.1, we merge different location slots and inverse slots into one slot type to use our training data most effectively and avoid redundant training. In Table 7, we provide an overview of the official slot types we cover with our models.

For the following slots, we could not extract enough training data to train machine learning models: per:charges, per:other_family, per:religion, org:date_dissolved, org:number_of_employees_members, org:political_religious_affiliation, org:shareholders, org:website, \{per, org, gpe\}:holds_shares_in. For those slots, our slot filling pipeline uses only the pattern matcher.

\section{Appendix B. Hyperparameters}

For tuning the CNN models, we performed grid-search over the following ranges of hyperparameters: filter width $\in\{3,5\}$, \# conv filters $\in\{100,300,1000,3000\}$, hidden units for relation extraction $\in\{100,300,1000\}$, hidden units for entity classification $\in\{25,100\}$. Other hyperparameters are the same for all models. For example, we use stochastic gradient descent with minibatches of size 10 and a learning rate of 0.1 . For regularization, we add a L2 penalty with a weight of 1e-5.

For the SVM models, we only tune $C$, the penalty of the error term. All the other parameters are set to the default parameters of the implementation. For example, the SVMs are trained with squared hinge loss and L2 regularization, the tolerance value for the stopping criteria is $1 \mathrm{e}-4$.

Table 8 shows the hyperparameters tuned on the development part of the slot filling relation classification benchmark dataset. The configuration files which we use to specify the hyperparameters for the different models are included in the code which we publish along with this paper. 


\section{ADEL \& SCHÜTZE}

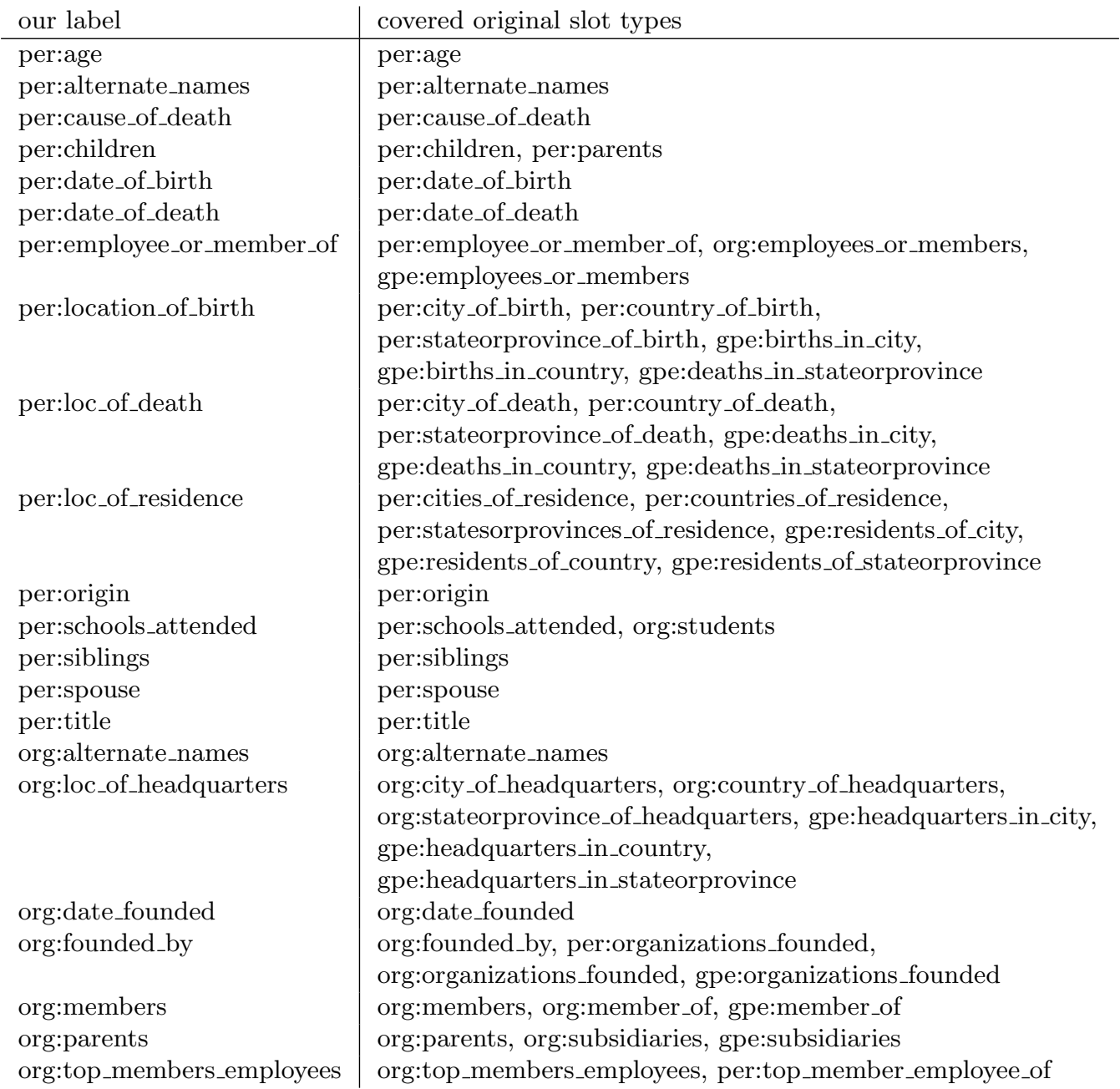

Table 7: Mapping showing which of our labels for classification covers which original slot filling slot types.

\section{References}

Adel, H., Roth, B., \& Schütze, H. (2016). Comparing convolutional neural networks to traditional models for slot filling. In Conference of the North American Chapter of the Association for Computational Linguistics: Human Language Technologies, pp. 828-838. Association for Computational Linguistics.

Adel, H., \& Schütze, H. (2015). CIS at TAC cold start 2015: Neural networks and coreference resolution for slot filling. In Text Analysis Conference. National Institute of 
Type-aware Convolutional Neural Networks for Slot Filling

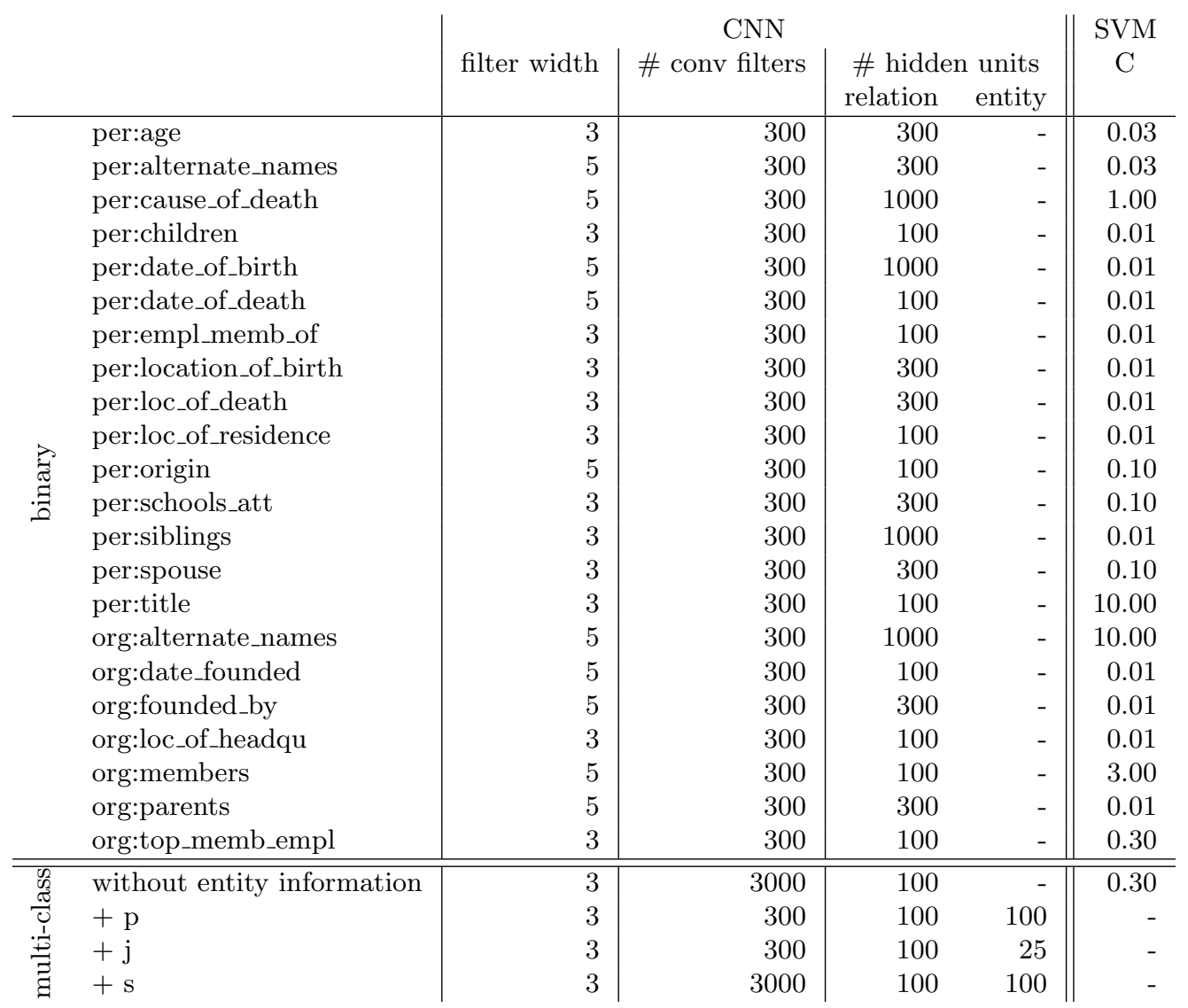

Table 8: Hyperparameters of CNNs and SVMs.

Standards and Technology.

Adel, H., \& Schütze, H. (2017). Global normalization of convolutional neural networks for joint entity and relation classification. In Conference on Empirical Methods in Natural Language Processing, pp. 1723-1729. Association for Computational Linguistics.

Angeli, G., Chaganty, A., Chang, A., Reschke, K., Tibshirani, J., Wu, J. Y., Bastani, O., Siilats, K., \& Manning, C. D. (2013). Stanford's 2013 KBP system. In Text Analysis Conference. National Institute of Standards and Technology.

Angeli, G., Gupta, S., Jose, M., Manning, C. D., Re, C., Tibshirani, J., Wu, J. Y., Wu, S., \& Zhang, C. (2014a). Stanford's 2014 slot filling systems. In Text Analysis Conference. National Institute of Standards and Technology.

Angeli, G., Tibshirani, J., Wu, J., \& Manning, C. D. (2014b). Combining distant and partial supervision for relation extraction. In Conference on Empirical Methods in Natural Language Processing, pp. 1556-1567. Association for Computational Linguistics. 
Angeli, G., Zhong, V., Chen, D., Chaganty, A., Bolton, J., Premkumar, M. J., Pasupat, P., Gupta, S., \& Manning, C. D. (2015). Bootstrapped self training for knowledge base population. In Text Analysis Conference. National Institute of Standards and Technology.

Bao, Z., Lu, Y., Tian, T., Liang, D., Zhao, Y., Li, Z., Qin, P., \& Xu, W. (2016). DoughnutPRIS at TAC KBP 2016. In Text Analysis Conference. National Institute of Standards and Technology.

Bollacker, K., Evans, C., Paritosh, P., Sturge, T., \& Taylor, J. (2008). Freebase: a collaboratively created graph database for structuring human knowledge. In ACM SIGMOD International Conference on Management of Data, pp. 1247-1250. ACM.

Byrne, L., Fenlon, C., \& Dunnion, J. (2013). UCD IIRG at TAC KBP 2013. In Text Analysis Conference. National Institute of Standards and Technology.

Byrne, L., Fenlon, C., \& Dunnion, J. (2014). UCD IIRG at TAC KBP 2014. In Text Analysis Conference. National Institute of Standards and Technology.

Chaganty, A. T., Paranjape, A. P., Liang, P., \& Manning, C. D. (2017). Importance sampling for unbiased on-demand evaluation of knowledge base population. In Conference on Empirical Methods in Natural Language Processing, pp. 1049-1059. Association for Computational Linguistics.

Chang, H.-S., Munir, A., Liu, A., Wei, J. T.-Z., Traylor, A., Nagesh, A., Monath, N., Verga, P., Strubell, E., \& McCallum, A. (2016). Extracting multilingual relations under limited resources: TAC 2016 cold-start KB construction and slot-filling using compositional universal schema. In Text Analysis Conference. National Institute of Standards and Technology.

Clueweb. Available online. http://lemurproject.org/clueweb12.

Cold-start-scoring-scripts. Available online. https://tac.nist.gov/2015/KBP/ ColdStart/tools/README.md.

Collobert, R., Weston, J., Bottou, L., Karlen, M., Kavukcuoglu, K., \& Kuksa, P. (2011). Natural language processing (almost) from scratch. Journal of Machine Learning Research, 12, 2493-2537.

Country-list. Available online. http://www.listof countriesof theworld.com.

Crammer, K., \& Singer, Y. (2001). On the algorithmic implementation of multiclass kernelbased vector machines. Journal of Machine Learning Research, 2, 265-292.

Das, R., Zaheer, M., Reddy, S., \& McCallum, A. (2017). Question answering on knowledge bases and text using universal schema and memory networks. In Annual Meeting of the Association for Computational Linguistics, pp. 358-365. Association for Computational Linguistics. 
Type-aware Convolutional Neural Networks for Slot Filling

dos Santos, C., Xiang, B., \& Zhou, B. (2015). Classifying relations by ranking with convolutional neural networks. In Annual Meeting of the Association for Computational Linguistics and International Joint Conference on Natural Language Processing of the Asian Federation of Natural Language Processing, pp. 626-634. Association for Computational Linguistics.

Fan, R.-E., Chang, K.-W., Hsieh, C.-J., Wang, X.-R., \& Lin, C.-J. (2008). LIBLINEAR: A library for large linear classification. Journal of Machine Learning Research, 9, $1871-1874$.

Ferschke, O., Zesch, T., \& Gurevych, I. (2011). Wikipedia revision toolkit: Efficiently accessing Wikipedia's edit history. In Annual Meeting of the Association for Computational Linguistics: Human Language Technologies: System Demonstrations, pp. 97-102. Association for Computational Linguistics.

Feys, M., Sterckx, L., Mertens, L., Deleu, J., Demeester, T., \& Develder, C. (2014). Ghent University-IBCN participation in TAC-KBP 2014 slot filling and cold start tasks. In Text Analysis Conference. National Institute of Standards and Technology.

Gonzàlez, E., Rodríguez, H., Turmo, J., Comas, P. R., Naderi, A., Ageno, A., Sapena, E., Vila, M., \& Martí, M. A. (2012). The TALP participation at TAC-KBP 2012. In Text Analysis Conference. National Institute of Standards and Technology.

Hong, Y., Wang, X., Chen, Y., Wang, J., Zhang, T., Zheng, J., Yu, D., Li, Q., Zhang, B., Wang, H., Pan, X., \& Ji, H. (2014). RPI_BLENDER TAC-KBP2014 knowledge base population system. In Text Analysis Conference. National Institute of Standards and Technology.

Huang, L., Sil, A., Ji, H., \& Florian, R. (2017). Improving slot filling performance with attentive neural networks on dependency structures. In Conference on Empirical Methods in Natural Language Processing, pp. 2578-2587. Association for Computational Linguistics.

Kalchbrenner, N., Grefenstette, E., \& Blunsom, P. (2014). A convolutional neural network for modelling sentences. In Annual Meeting of the Association for Computational Linguistics, pp. 655-665. Association for Computational Linguistics.

Kisiel, B., Betteridge, J., Gardner, M., Krishnamurthy, J., Nakashole, N., Platanios, E. A., Ritter, A., Samadi, M., Saparov, A., Talukdar, P., Wijaya, D., \& Mitchell, T. (2014). CMUML system for KBP 2014 slot filling. In Text Analysis Conference. National Institute of Standards and Technology.

Kisiel, B., McDowell, B., Gardner, M., Nakashole, N., Platanios, E. A., Saparov, A., Srivastava, S., Wijaya, D., \& Mitchell, T. (2015). CMUML system for KBP 2015 cold start slot filling. In Text Analysis Conference. National Institute of Standards and Technology. 
Lample, G., Ballesteros, M., Subramanian, S., Kawakami, K., \& Dyer, C. (2016). Neural architectures for named entity recognition. In Conference of the North American Chapter of the Association for Computational Linguistics: Human Language Technologies, pp. 260-270. Association for Computational Linguistics.

Lehmann, J., Isele, R., Jakob, M., Jentzsch, A., Kontokostas, D., Mendes, P. N., Hellmann, S., Morsey, M., van Kleef, P., Auer, S., \& Bizer, C. (2015). DBpedia - A large-scale, multilingual knowledge base extracted from wikipedia. Semantic Web, 6(2), 167-195.

Li, Y., Chen, S., Zhou, Z., Yin, J., Luo, H., Hong, L., Xu, W., Chen, G., \& Guo, J. (2012). PRIS at TAC2012 KBP track. In Text Analysis Conference. National Institute of Standards and Technology.

LinearSVC. Available online. http://scikit-learn.org/stable/modules/generated/ sklearn.svm.LinearSVC.html.

Manning, C., Surdeanu, M., Bauer, J., Finkel, J., Bethard, S., \& McClosky, D. (2014). The Stanford CoreNLP natural language processing toolkit. In Annual Meeting of the Association for Computational Linguistics: System Demonstrations, pp. 55-60. Association for Computational Linguistics.

Mendes, P. N., Jakob, M., \& Bizer, C. (2012). DBpedia for NLP: A multilingual crossdomain knowledge base. In International Conference on Language Resources and Evaluation, pp. 1813-1817. European Language Resources Association.

Mikolov, T., Chen, K., Corrado, G., \& Dean, J. (2013). Efficient estimation of word representations in vector space. In International Conference on Learning Representations Workshop.

Min, B., \& Grishman, R. (2012). Challenges in the knowledge base population slot filling task. In International Conference on Language Resources and Evaluation, pp. 11371142. European Language Resources Association.

Mintz, M., Bills, S., Snow, R., \& Jurafsky, D. (2009). Distant supervision for relation extraction without labeled data. In Annual Meeting of the Association for Computational Linguistics and International Joint Conference on Natural Language Processing of the Asian Federation of Natural Language Processing, pp. 1003-1011. Association for Computational Linguistics.

Miwa, M., \& Bansal, M. (2016). End-to-end relation extraction using LSTMs on sequences and tree structures. In Annual Meeting of the Association for Computational Linguistics, pp. 1105-1116. Association for Computational Linguistics.

Miwa, M., \& Sasaki, Y. (2014). Modeling joint entity and relation extraction with table representation. In Conference on Empirical Methods in Natural Language Processing, pp. 1858-1869. Association for Computational Linguistics. 
Nguyen, T. H., He, Y., Pershina, M., Li, X., \& Grishman, R. (2014). New York University 2014 knowledge base population systems. In Text Analysis Conference. National Institute of Standards and Technology.

Nicknames. Available online. http://usefulenglish.ru/vocabulary/mens-names, http://usefulenglish.ru/vocabulary/womens-names.

Ounis, I., Amati, G., Plachouras, V., He, B., Macdonald, C., \& Lioma, C. (2006). Terrier: A high performance and scalable information retrieval platform. In SIGIR Workshop on Open Source Information Retrieval, pp. 18-24. ACM.

Pawar, S., Bhattacharyya, P., \& Palshikar, G. (2017). End-to-end relation extraction using neural networks and markov logic networks. In Conference of the European Chapter of the Association for Computational Linguistics, pp. 818-827. Association for Computational Linguistics.

Piccinno, F., \& Ferragina, P. (2014). From TagME to WAT: A new entity annotator. In International workshop on Entity recognition $\&$ disambiguation, pp. 55-62. Association for Computing Machinery.

Pink, G., Nothman, J., \& Curran, J. R. (2014). Analysing recall loss in named entity slot filling. In Conference on Empirical Methods in Natural Language Processing, pp. 820-830. Association for Computational Linguistics.

Qin, P., Ma, C., Jia, Y., Wang, W., Zhang, Z., Bao, Z., Xu, W., \& Guo, J. (2015). BUPT_PRIS at TAC KBP 2015. In Text Analysis Conference. National Institute of Standards and Technology.

Rabiner, L. R. (1990). A tutorial on hidden Markov models and selected applications in speech recognition. In Waibel, A., \& Lee, K.-F. (Eds.), Readings in Speech Recognition, pp. 267-296. Morgan Kaufmann Publishers Inc.

Roth, B., Barth, T., Wiegand, M., Singh, M., \& Klakow, D. (2013). Effective slot filling based on shallow distant supervision methods. In Text Analysis Conference. National Institute of Standards and Technology.

Roth, B., Monath, N., Belanger, D., Strubell, E., Verga, P., \& McCallum, A. (2015). Building knowledge bases with universal schema: Cold start and slot-filling approaches. In Text Analysis Conference. National Institute of Standards and Technology.

Roth, D., \& Yih, S. W.-t. (2007). Global Inference for Entity and Relation Identification via a Linear Programming Formulation, pp. 553-580. MIT Press.

Slot-descriptions. Available online. http://surdeanu.info/kbp2014/TAC_KBP_2014_ Slot_Descriptions_V1.1.pdf.

Slot-filling. Available online. https://tac.nist.gov/2017/KBP/ColdStart/index.html. 
Soderland, S., Hawkins, N., Gilmer, J., \& Weld, D. S. (2015). Combining open IE and distant supervision for KBP slot filling. In Text Analysis Conference. National Institute of Standards and Technology.

State-list. Available online. http://en.wikipedia.org/wiki/List_of_U.S._state_ abbreviations.

Sterckx, L., Demeester, T., Deleu, J., \& Develder, C. (2015). Ghent University - IBCN participation in the TAC KBP 2015 cold start slot filling task. In Text Analysis Conference. National Institute of Standards and Technology.

Surdeanu, M. (2013). Overview of the TAC2013 knowledge base population evaluation: English slot filling and temporal slot filling. In Text Analysis Conference. National Institute of Standards and Technology.

Surdeanu, M., \& Ji, H. (2014). Overview of the english slot filling track at the TAC2014 knowledge base population evaluation. In Text Analysis Conference. National Institute of Standards and Technology.

Surdeanu, M., Tibshirani, J., Nallapati, R., \& Manning, C. D. (2012). Multi-instance multilabel learning for relation extraction. In Joint Conference on Empirical Methods in Natural Language Processing and Computational Natural Language Learning, pp. 455-465. Association for Computational Linguistics.

Vu, N. T., Adel, H., Gupta, P., \& Schütze, H. (2016). Combining recurrent and convolutional neural networks for relation classification. In Conference of the North American Chapter of the Association for Computational Linguistics: Human Language Technologies, pp. 534-539. Association for Computational Linguistics.

Wazalwar, A., Khot, T., Zhang, C., Ré, C., Shavlik, J., \& Natarajan, S. (2014). TAC KBP 2014 : English slot filling track DeepDive with expert advice. In Text Analysis Conference. National Institute of Standards and Technology.

Wikipedia. Available online. https://en.wikipedia.org.

Yaghoobzadeh, Y., Adel, H., \& Schütze, H. (2017). Noise mitigation for neural entity typing and relation extraction. In Conference of the European Chapter of the Association for Computational Linguistics, pp. 1183-1194. Association for Computational Linguistics.

Yang, B., Nakashole, N., Kisiel, B., Platanios, E. A., Saparov, A., Srivastava, S., Wijaya, D., \& Mitchell, T. (2016). CMUML micro-reader system for KBP 2016 cold start slot filling, event nugget detection, and event argument linking. In Text Analysis Conference. National Institute of Standards and Technology.

Yao, L., Riedel, S., \& McCallum, A. (2010). Collective cross-document relation extraction without labelled data. In Conference on Empirical Methods in Natural Language Processing, pp. 1013-1023. Association for Computational Linguistics. 
Yu, D., \& Ji, H. (2016). Unsupervised person slot filling based on graph mining. In Annual Meeting of the Association for Computational Linguistics, pp. 44-53. Association for Computational Linguistics.

Yu, D., Pan, X., Zhang, B., Huang, L., Lu, D., Whitehead, S., \& Ji, H. (2016). RPI_BLENDER TAC-KBP2016 system description. In Text Analysis Conference. National Institute of Standards and Technology.

Zeng, D., Liu, K., Lai, S., Zhou, G., \& Zhao, J. (2014). Relation classification via convolutional deep neural network. In International Conference on Computational Linguistics, pp. 2335-2344. Association for Computational Linguistics.

Zhang, C., Xu, W., Qin, P., Qi, A., Liu, Y., Wang, X., \& Qunluo (2014). The PRIS systems at slot filling and entity linking 2014. In Text Analysis Conference. National Institute of Standards and Technology.

Zhang, Y., Chaganty, A., Paranjape, A., Chen, D., Bolton, J., Qi, P., \& Manning, C. D. (2016). Stanford at TAC KBP 2016: Sealing pipeline leaks and understanding Chinese. In Text Analysis Conference. National Institute of Standards and Technology.

Zhang, Y., Zhong, V., Chen, D., Angeli, G., \& Manning, C. D. (2017). Position-aware attention and supervised data improve slot filling. In Conference on Empirical Methods in Natural Language Processing, pp. 35-45. Association for Computational Linguistics. 